\title{
Ly6C ${ }^{10}$ monocytes drive immunosuppression and confer resistance to anti-VEGFR2 cancer therapy
}

\author{
Keehoon Jung, ${ }^{1}$ Takahiro Heishi, ${ }^{1}$ Omar F. Khan, ${ }^{2}$ Piotr S. Kowalski, ${ }^{2}$ Joao Incio, ${ }^{1}$ Nuh N. Rahbari, ${ }^{1}$ Euiheon Chung, ${ }^{1}$ \\ Jeffrey W. Clark, ${ }^{3}$ Christopher G. Willett, ${ }^{4}$ Andrew D. Luster, ${ }^{5}$ Seok Hyun Yun, ${ }^{6,7}$ Robert Langer, ${ }^{2,7}$ Daniel G. Anderson, ${ }^{2,7}$ \\ Timothy P. Padera, ${ }^{1}$ Rakesh K. Jain, ${ }^{1}$ and Dai Fukumura' \\ 'Edwin L. Steele Laboratories for Tumor Biology, Department of Radiation Oncology, Harvard Medical School and Massachusetts Ceneral Hospital, Boston, Massachusetts, USA. ${ }^{2}$ David H. Koch Institute for \\ Integrative Cancer Research, Department of Chemical Engineering, Massachusetts Institute of Technology (MIT), Cambridge, Massachusetts, USA. ${ }^{3}$ Department of Hematology/Oncology, Harvard Medical \\ School and Massachusetts General Hospital, Boston, Massachusetts, USA. ${ }^{4}$ Duke University Medical Center, Durham, North Carolina, USA. ${ }^{5}$ Center for Immunology and Inflammatory Diseases, Division of \\ Rheumatology, Allergy and Immunology, Harvard Medical School and Massachusetts General Hospital, Boston, Massachusetts, USA. ${ }^{6}$ Wellman Center for Photomedicine, Department of Dermatology, \\ Harvard Medical School and Massachusetts General Hospital, Boston, Massachusetts, USA. ${ }^{7}$ Harvard-MIT Division of Health Sciences and Technology, Cambridge, Massachusetts, USA
}

Current anti-VEGF therapies for colorectal cancer (CRC) provide limited survival benefit, as tumors rapidly develop resistance to these agents. Here, we have uncovered an immunosuppressive role for nonclassical Ly6C ${ }^{10}$ monocytes that mediates resistance to anti-VEGFR2 treatment. We found that the chemokine CX3CL1 was upregulated in both human and murine tumors following VEGF signaling blockade, resulting in recruitment of CX3CR1+Ly6C ${ }^{10}$ monocytes into the tumor. We also found that treatment with VECFA reduced expression of CX3CL1 in endothelial cells in vitro. Intravital microscopy revealed that CX3CR1 is critical for Ly6 $\mathrm{C}^{10}$ monocyte transmigration across the endothelium in murine CRC tumors. Moreover, Ly6C $\mathrm{C}^{\circ}$ monocytes recruit Ly6C+ neutrophils via CXCL5 and produce IL-10, which inhibits adaptive immunity. Preventing Ly6Clo monocyte or Ly6C+ neutrophil infiltration into tumors enhanced inhibition of tumor growth with anti-VECFR2 therapy. Furthermore, a gene therapy using a nanoparticle formulated with an siRNA against CX3CL1 reduced Ly6C ${ }^{10}$ monocyte recruitment and improved outcome of anti-VECFR2 therapy in mouse CRCs. Our study unveils an immunosuppressive function of $\mathrm{Ly}_{6 \mathrm{C}} \mathrm{C}^{\circ}$ monocytes that, to our knowledge, has yet to be reported in any context. We also reveal molecular mechanisms underlying antiangiogenic treatment resistance, suggesting potential immunomodulatory strategies to enhance the long-term clinical outcome of anti-VEGF therapies.

\section{Introduction}

Angiogenesis is a hallmark of cancer (1). The VEGF signaling pathway is a key component of pathological angiogenesis in most cancers (2-5). To date, 10 anti-VEGF drugs have been approved by the FDA to treat various solid tumors, starting with metastatic colorectal cancer (CRC) in 2004 (1). However, the survival benefit from these drugs is modest, as tumors develop resistance to these agents $(3,4$, 6-13). The mechanisms of resistance remain far from understood.

The immune microenvironment has drawn great interest for its strong influence on tumor growth (7, 8, 10, 12, 14-22). Emerging data show that the immune system also plays a critical role in refractoriness to antiangiogenic therapy $(13,23,24)$. Among various types of leukocytes, a growing body of evidence suggests that immunosuppressive innate immune cells contribute to this

Authorship note: R.K. Jain and D. Fukumura are co-senior authors.

Conflict of interest: R.K. Jain received consultant fees from Ophthotech Corp., SPARC,

SynDevRx, and XTuit. R.K. Jain owns equity in Enlight, Ophthotech Corp., SynDevRx, and XTuit and serves on the Board of Directors of XTuit and the Boards of Trustees of Tekla Healthcare Investors, Tekla Life Sciences Investors, Tekla Healthcare Opportunities Fund, and Tekla World Healthcare Fund. No reagents or funding from these companies was used in this work.

Submitted: February 1, 2017; Accepted: May 19, 2017

Reference information: J Clin Invest. 2017;127(8):3039-3051.

https://doi.org/10.1172/JCI93182. resistance in addition to cancer cell immune evasion $(6,25-27)$. However, these myeloid cells are a collection of diverse subsets of CD11b+ monocytic and granulocytic cells (27-30), which have often been studied together rather than as clearly defined subpopulations. Furthermore, Ly6 $\mathrm{C}^{\text {lo }}$ monocytes, also known as nonclassical monocytes, have not yet been clearly characterized or extensively investigated in the context of anti-VEGF cancer therapy. Particularly, it is not known whether Ly6 $\mathrm{C}^{\text {lo }}$ monocytes are capable of promoting immunosuppression in any context.

Moreover, mechanistic studies on the role of chemokines/ chemokine receptors in each specific subpopulation of innate immune cells in cancers have not been conducted, even though the importance of chemokines in leukocyte trafficking has long been widely accepted $(31,32)$. The lack of suitable methods for in vivo longitudinal cellular-level monitoring of leukocytes in CRCs of small animal models has limited previous efforts to elucidate the highly dynamic immune microenvironment. Thus, the role and kinetics of specific subsets of innate immune cells in conferring resistance to anti-VEGF therapy are not known.

Here, we uncover a function of Ly6 $\mathrm{C}^{\mathrm{lo}}$ monocytes - immunosuppression - and reveal that they instigate a cascade of immunomodulatory events in response to anti-VEGF therapy. We found that anti-VEGF therapy upregulates CX3CL1 expression, which facilitates CX3CR1-dependent infiltration of Ly6 $\mathrm{C}^{\text {lo }}$ monocytes. 

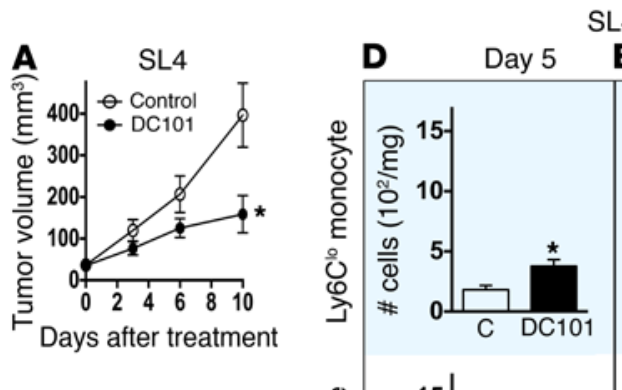

E Day 12

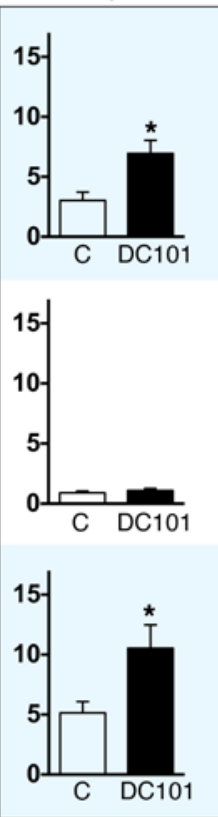

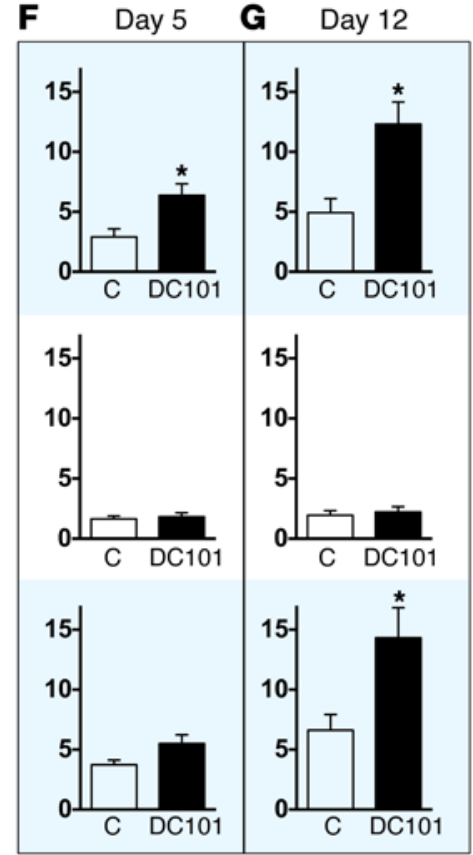
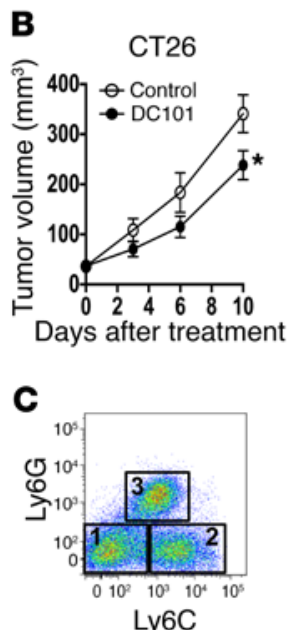

Ly6C
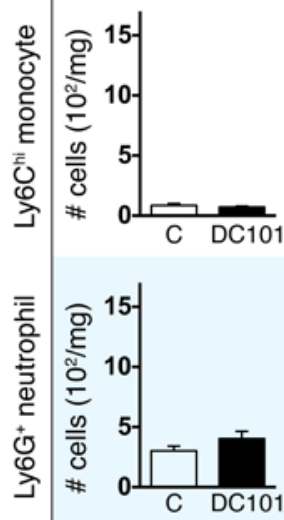

Figure 1. Anti-VEGFR2 therapy facilitates early infiltration of Ly6C $\mathbf{C}^{10}$ monocytes into tumors. (A and B) Tumor volume was measured using a highfrequency ultrasound imaging system for orthotopically grown syngeneic SL4 tumors in C57BL/6 mice (A) and CT26 tumors in BALB/c mice (B). Tumors were treated with either control rat IgG (control) or monoclonal anti-VEGFR2 antibody DC101 (40 mg/kg, every 3 days). $n=8 /$ group. (C) A representative flow cytometry plot depicting the 3 different subsets of myeloid populations. 1, Ly6C ${ }^{\text {lo }}$ monocyte; 2, Ly6C ${ }^{\text {hi }}$ monocyte; 3, Ly6C ${ }^{+}$neutrophil. C57BL/6 WT mice bearing SL4 tumors were treated with DC101, and immune cells in the tumor infiltrate were analyzed on day 5 by flow cytometry. Gated on CD45+Lin

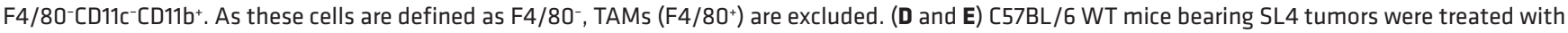
either control rat IgG (C) or DC101. Each subset of myeloid cells in tumor infiltrate was analyzed on day 5 (D) and day 12 (E) by flow cytometry. Top row, Ly6 $\mathrm{C}^{10}$ monocyte; center row, Ly6C $\mathrm{C}^{\text {hi }}$ monocyte; bottom row, Ly6C+ $\mathrm{C}^{+}$eutrophil. $n=8 /$ group. (F and $\left.\mathbf{G}\right)$ BALB/c WT mice bearing CT26 tumors were divided into 2 different treatment groups (control, DC101), and the myeloid cell subsets in the tumor infiltrate were analyzed on day 5 (F) and day 12 (G) by flow cytometry. The graphs depict the absolute number of cells per mg of tumor tissue. $n=8$ /group. Data are represented as mean $\pm S E M$. ${ }^{*} P<0.05$ versus control, 2-tailed $t$ tests. Data are representative of 4 ( $\mathbf{A}$ and $\mathbf{B}$ ) or 3 (D-C) independent experiments.

These CX3CR $1^{+}$Ly6 $\mathrm{C}^{\text {lo }}$ monocytes produce CXCL5 to recruit neutrophils to the tumors and create an immunosuppressive microenvironment. Furthermore, we developed a gene therapy method to target CX3CL1, which significantly $(P<0.05)$ improved the efficacy of anti-VEGF cancer therapy by inhibiting CX3CR1 ${ }^{+}$Ly $6 \mathrm{C}^{\text {lo }}$ monocyte infiltration. These findings, based on multimodal approaches, including genetic ablation of chemokine receptors and intravital multiphoton microscopy, offer a mechanistic basis to develop novel and efficient immunotherapeutic strategies to treat solid cancers.

\section{Results}

Anti-VEGFR2 therapy induces accumulation of monocytes and neutrophils in CRCs. To examine the role of the immune microenvironment in CRCs, we utilized 2 syngeneic murine CRC models - SL4 and CT26 - orthotopically implanted in C57BL/6 and $\mathrm{BALB} / \mathrm{c}$ mice, respectively. We also studied spontaneous rectal tumors in conditional Apc mutant mice (Apct/fl Ad-Cre) (33). We used DC101, a monoclonal antibody against VEGFR2, to inhibit angiogenesis (34). We observed vessel regression and increased hypoxia on days 5 and 12 after DC101 treatment compared with the control, while there were no observable changes in microvessel density (MVD) or hypoxia on day 2 (Supplemental Figure 1, A-D; supplemental material available online with this article; https://doi.org/10.1172/JCI93182DS1). Interestingly, there were differences in responses to DC101 between the 2 orthotopic CRC models, with SL4 being more sensitive to antiangiogenic therapy than CT26. After DC101 monotherapy, the SL4 tumor size was approximately $40 \%$ of that of the control, while CT26 tumor size was approximately $70 \%$ (Figure $1, \mathrm{~A}$ and $\mathrm{B}$ ).

Consistent with published data from anti-VEGF therapies in other tumor models (23), we found a significant increase in $\mathrm{CD}_{11} \mathrm{~b}^{+} \mathrm{Gr} 1^{+}$myeloid cells in our CRC models after DC101 treatment (Supplemental Figure 2A). However, the $\mathrm{CD}_{11} \mathrm{~b}^{+} \mathrm{Gr} 1^{+}$cells represent a heterogeneous mixture of monocytic and granulocytic myeloid cells $(28-30,35)$. Although separate analyses for the different subpopulations of myeloid cells are essential for better understanding of the biology, the definition of $\mathrm{CD} 11 \mathrm{~b}^{+} \mathrm{Gr} 1^{+}$cell subpopulations using surface markers has been ambiguous among research groups. Previous studies have focused on $\mathrm{Gr}^{\text {hi }}{ }^{\left(\mathrm{Ly} 6 \mathrm{C}^{\text {hi }} \text { or }\right.}$ Ly $\left.6 G^{+}\right)$myeloid cells $(26,28,35-43)$. In this study, we clearly discriminate between $\mathrm{Ly} 6 \mathrm{C}^{\mathrm{hi}}$ and $\mathrm{Ly} 6 \mathrm{G}^{+}$myeloid cell subsets based 

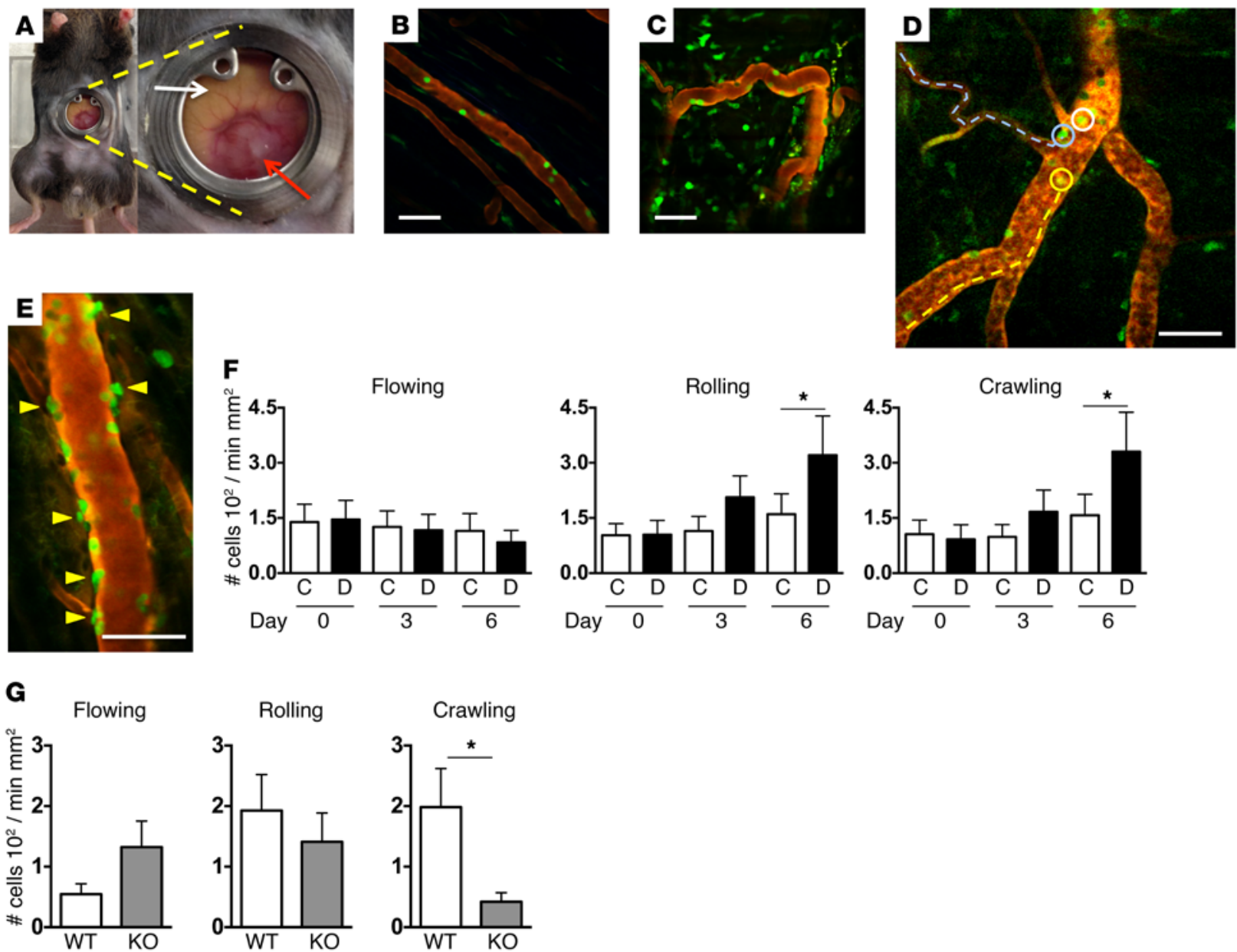

Figure 2. Ly6C ${ }^{10}$ monocytes require CX3CR1 to infiltrate into tumors during anti-VEGFR2 therapy. (A) Abdominal imaging window on a live mouse bearing syngeneic SL4 CRC (red arrow) in the cecum (white arrow). (B and C) Images of crawling CX3CR1+ leukocytes (green) inside the postcapillary venule (red, TRITC-dextran) in a normal cecum (B) and in the tumor (C) of a Cx3cr19fp/+ mouse. Ly6C ${ }^{10}$ monocytes are labeled with ECFP (green). Ly6C ${ }^{10}$ monocytes are also observed in the tumor (C). (D) Snapshot image taken at 8 seconds of Supplemental Video 1 showing flowing (gray), rolling (yellow), and crawling (white) CX3CR1 ${ }^{+}$Ly6C $C^{10}$ monocytes inside the blood vessels in an SL4 tumor. (E) Snapshot image showing CX3CR $1^{+}$Ly6C $C^{10}$ monocytes undergoing extravasation in an SL4 tumor. Red, TRITC-dextran (blood vessels). (F) Flux of flowing, rolling, and crawling CX3CR1+Ly6C ${ }^{10}$ monocytes in blood circulation in SL4 tumor-bearing $C \times 3 \mathrm{cr} 19 f \mathrm{fp} /+$ mice treated with either control rat IgC (C) or DC101 (D). (C) Flux of flowing, rolling, and crawling Ly6 $\mathrm{C}^{10}$ monocytes in blood circulation in SL4 tumor-bearing C57BL/6 WT mice at 5 days after DC101 treatment. Ly6C ${ }^{10}$ monocytes were isolated from C57BL/6 WT or $\left[\times 3 \mathrm{cr}^{-1 /-}\right.$ mice (KO), fluorescently labeled, and adoptively transferred into DC101-treated SL4 tumor-bearing C57BL/6 WT animals. $n=7 /$ group. Data are represented as mean \pm SEM. ${ }^{*} P<0.05$, 2-tailed $t$ tests. Data are representative of 3 independent experiments (F and $\left.\mathbf{G}\right)$. Scale bars: $100 \mu \mathrm{m}(\mathbf{B}-\mathbf{E})$.

on their immunophenotype (i.e., Ly6 $\mathrm{C}^{\text {hi }}$ monocytes and $\mathrm{Ly} 6 \mathrm{G}^{+}$ neutrophils, respectively) (Figure 1C) (gated on $\mathrm{CD}^{4} 5^{+} \mathrm{Lin}^{-} \mathrm{F} 4 / 80$

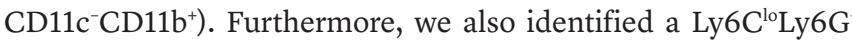
population, Ly $6 \mathrm{C}^{\mathrm{lo}}$ monocytes (Figure 1C), which have not been reported in tumors after anti-VEGF therapy. These cells display a high level of CX3CR1, while Ly $6 \mathrm{C}^{\text {hi }}$ monocytes and Ly6 $6 \mathrm{G}^{+}$neutrophils (hereafter referred to as neutrophils) express CCR2 and CXCR2, respectively $(25,39,44)$ (Supplemental Figure 2B).

Anti-VEGFR2 therapy facilitates early infiltration of $L y 6 C^{l o}$ monocytes into tumors. Among the 3 myeloid cell subpopulations found in SL4 tumors 5 days after DC101 treatment, there was a selective increase in Ly6 $\mathrm{C}^{\text {lo }}$ monocytes $(380 \pm 50$ cells $/ \mathrm{mg}$ ) compared with the IgG control ( $180 \pm 40$ cells $/ \mathrm{mg}$ ), while the other 2 myeloid cell subsets did not change significantly (Figure 1D). On day 12, we observed a further increase in Ly $6 \mathrm{C}^{\text {lo }}$ monocytes $(300 \pm 70$ cells/ $\mathrm{mg}$ in control vs. $700 \pm 110$ cells/mg in DC101) and also a signifi- cant increase in neutrophils $(510 \pm 100$ cells/mg in control vs. 1050 \pm 190 cells/mg in DC101) (Figure 1E). Ly6 $\mathrm{C}^{\text {hi }}$ monocytes remained at similar levels between treatment groups on day 12 (Figure 1E). Given that the average size of the control group tumors harvested on day 5 and that of the DC101 group on day 12 were similar, the difference in the number of recruited $\mathrm{Ly} 6 \mathrm{C}^{\mathrm{lo}}$ monocytes between 2 treatment groups is attributed to the treatment (IgG vs. DC101), not to the stage of tumor progression. In CT26 tumors, DC101 treatment showed similar kinetic response of Ly6 $\mathrm{C}^{\text {lo }}$ monocytes and neutrophils (Figure 1, F and G). In spontaneous rectal tumors in conditional Apc mutant mice, we also observed Ly6 $\mathrm{C}^{\text {lo }}$ monocytes infiltrating prior to neutrophils (Supplemental Figure 3).

Ly6C $C^{l o}$ monocytes progressively infiltrate into tumors over the course of anti-VEGFR 2 treatment. To investigate the dynamic infiltration of Ly $6 \mathrm{C}^{\mathrm{lo}}$ monocytes into orthotopic CRC tumors during antiangiogenic treatment, we surgically implanted an abdominal 

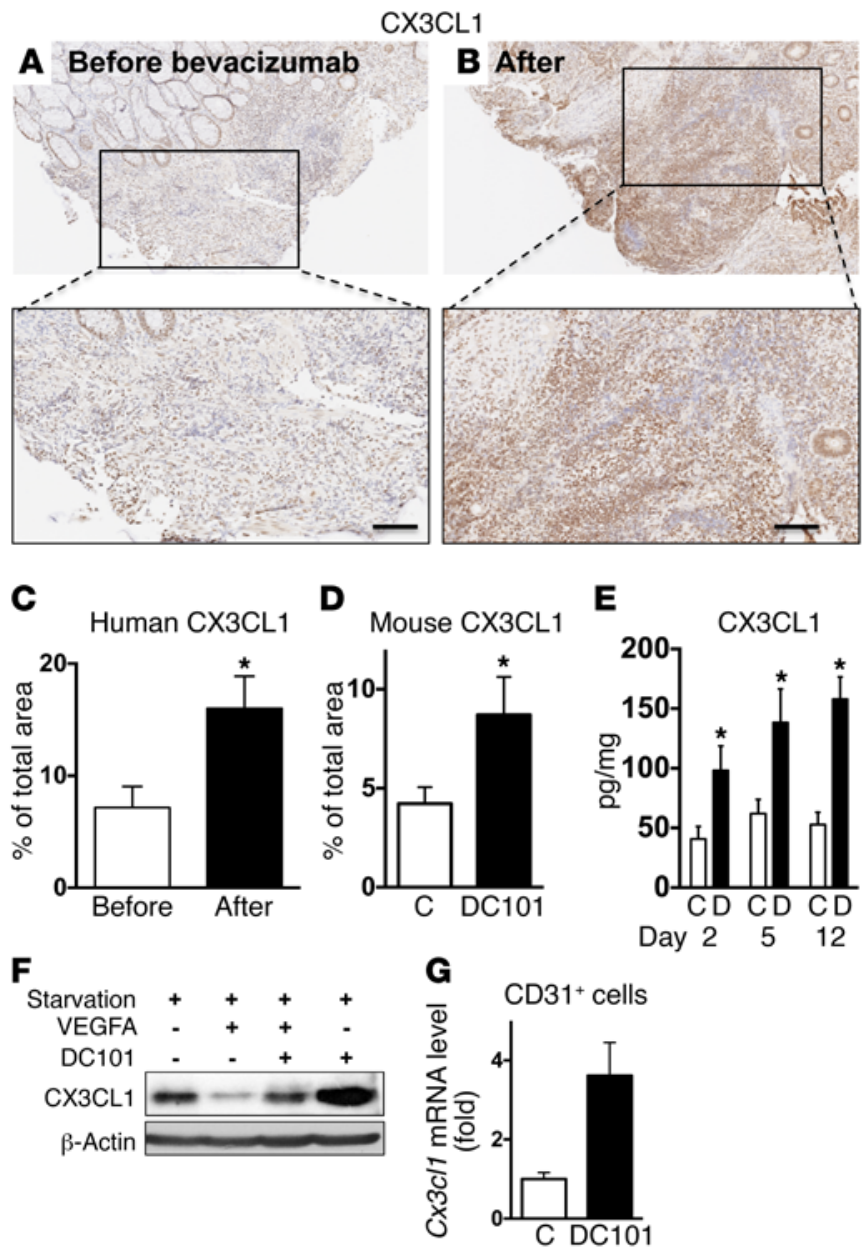

imaging window. (Figure 2A and Supplemental Figure 4). Unlike in previous windows $(45,46)$, the coverslip was removable for aspirating accumulated fluid, allowing clearer imaging of the gut for over 4 weeks using a custom-built video-rate multiphoton microscope (47) (Figure 2, A-F, and Supplemental Figure 4B). Ly $6 \mathrm{C}^{\text {lo }}$ monocytes expressed a high amount of CX3CR1 compared with other myeloid cell subsets (Supplemental Figure 2B), and CX3CR $1^{+}$cells in the bloodstream were predominantly monocytic (48-50). Thus, we used $C \times 3 c r 1^{g f p /+}$ knockin mice implanted with SL4 tumors in which Ly6 $\mathrm{C}^{\text {lo }}$ monocytes express EGFP (50-52). In animals treated with DC101, we frequently observed EGFP monocytes freely flowing in the blood that then began to interact with the vessel wall - either rolling or crawling (Figure 2, D and F, and Supplemental Figure 5; Supplemental Video 1). Some of the crawling cells subsequently extravasated from the blood vessel (Figure 2E). We found that DC101 significantly increased the number of rolling and crawling $\mathrm{EGFP}^{+} \mathrm{Ly} 6 \mathrm{C}^{\mathrm{lo}}$ monocytes compared with the control on day 6 (Figure $2 \mathrm{~F}$ ). There was no significant change in the leukocyte-endothelial cell interaction in the control tumors over time (Figure $2 \mathrm{~F}$ ). These results show that the blood serves as the source of tumor-infiltrated Ly $6 \mathrm{C}^{\text {lo }}$ monocytes, rather than local proliferation of $\mathrm{Ly}^{6} \mathrm{C}^{\mathrm{lo}}$ monocytes in the tumor parenchyma, that interact with the tumor vessels and subsequently transmigrate across the endothelium in a time-dependent manner during antiangiogenic treatment.
Figure 3. Blockade of VEGF/VEGFR2 signaling upregulates CX3CL1 in both human and mouse CRCs. (A and B) Representative images showing CX3CL1 (fractalkine) expression in human tissue sections from patients with rectal carcinomas (total 7 pairs) before (A) and after (B) bevacizumab treatment. Scale bar: $100 \mu \mathrm{m}$. (C) Averaged percentage of $\mathrm{CX} 3 \mathrm{CL}^{+}$area out of total area from tissue sections of 7 rectal cancer patients before and after bevacizumab treatment. $n=7 /$ group. ${ }^{*} P<0.05$ versus before, 2-tailed $t$ tests. (D) CX3CL1+ area percentage of total viable area from SL4 tumors treated with either control rat IgC (C) or DC101 analyzed on day 12. $n=7 /$ group. ${ }^{*} P<0.05$ versus control, 2 -tailed $t$ tests. (E) CX3CL1 protein levels measured from tissue lysates of tumors treated with either control rat lgG (C) or DC101 (D). $n=5 /$ group. ${ }^{*} P<0.05$ versus control, 2 -tailed $t$ tests. (F) Western blot analysis of $\mathrm{CX} 3 \mathrm{CL} 1$ protein expression in endothelial cells in vitro. Serum-starved endothelial cells were treated with either recombinant VEGFA protein, DC101, or VEGFA protein plus DC101, and CX3CL1 protein levels were measured from cell lysates. The blockade of VEGF/VEGFR2 signaling stimulates upregulation of CX3CL1 in endothelial cells. Three independent experiments showed similar findings. (C) BALB/C WT mice bearing orthotopically grown syngeneic CT26 CRCs were treated with either control rat IgC (C) or DC101 (D). Relative $[x 3 c / 1$ mRNA expression levels in endothelial cells isolated from CT26 tumors were determined on day 2 after treatment by quantitative real-time PCR, normalized against Gapdh. $n=8$ /group. Data are represented as mean \pm SEM.

Ly6C $C^{\text {lo }}$ monocytes require CX3CR1 to infiltrate into tumors. While the CX3CL1/CX3CR1 axis has long been known to be important in chemotaxis $(31,53)$, it has now become questionable whether CX3CR1 is important for the process of Ly6 $\mathrm{C}^{\text {lo }}$ monocyte recruitment or not (50). There have also been reports suggesting that CX3CR1 deficiency influences the survival of monocytes (54, 55). To determine whether CX3CR1 is critical for Ly $6 \mathrm{C}^{\mathrm{lo}}$ monocyte transmigration across the endothelium, we isolated $\mathrm{Ly} 6 \mathrm{C}^{\mathrm{lo}}$ monocytes from both WT and $\mathrm{C} x 3 \mathrm{Cr}^{-/-}$mice that were fluorescently labeled and adoptively transferred into DC101-treated WT mice bearing CRC tumors (Supplemental Videos 2 and 3). Measured by intravital microscopy, there was a significant decrease in the number of crawling Ly6 $\mathrm{C}^{\text {lo }}$ monocytes isolated from $\mathrm{Cx} 3 \mathrm{crl}^{-/-}$mice compared with those isolated from WT mice (Figure $2 \mathrm{G}$ ). These observations suggest that CX3CR1 plays an important role in chemotaxis-driven transmigration of Ly6 $\mathrm{C}^{\text {lo }}$ monocytes, especially in the process of rolling-crawling transition.

Blockade of VEGF/VEGFR2 signaling upregulates CX3CL1 in both human and mouse CRCs. Next, we examined which factors contribute to the CX3CR1-dependent attraction of Ly6C lo monocytes after DC101 treatment. CX3CL1, also known as fractalkine, is the only known ligand for CX3CR1 $(31,53)$. Immunohistochemistry showed that CX3CL1 is dramatically upregulated after DC101 treatment (Figure 3). Furthermore, biopsies of rectal carcinomas from patients before and after bevacizumab treatment (56, 57) also showed a significant increase in CX3CL1 expression after bevacizumab treatment (Figure 3, A-C).

By measuring protein levels, we found an increase in CX3CL1 in tumor tissues from day 2 onwards after DC101 treatment (Figure 3, D and E). Furthermore, we found increased CX3CL1 expression in endothelial cells isolated from tumors treated with DC101 (Figure 3G), consistent with published data showing endothelial cells as a source of $\operatorname{CX} 3 \operatorname{CL} 1(31,58)$, while there was no change in CX3CL1 expression in nonendothelial cells (Supplemental Figure 8I). Interestingly, endothelial cells treated with recombinant VEGFA protein to activate VEGF/VEGFR2 signaling in vitro 
A

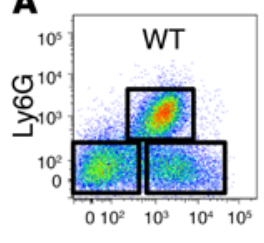

B

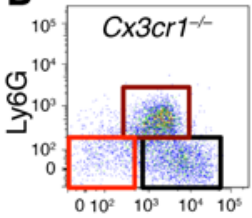

C

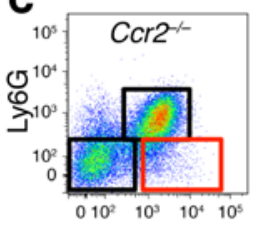

D

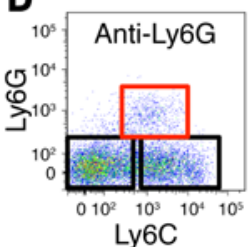

E
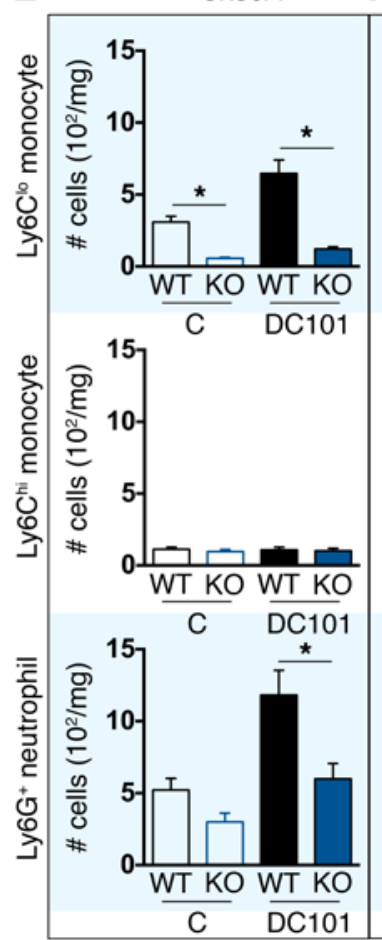

$\mathbf{F}$
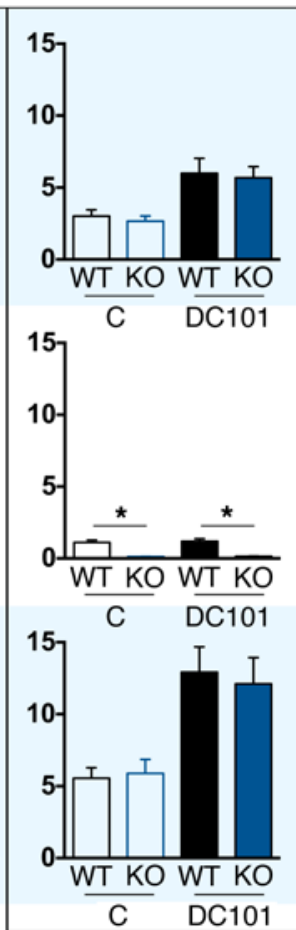

G Anti-Ly6G Ab

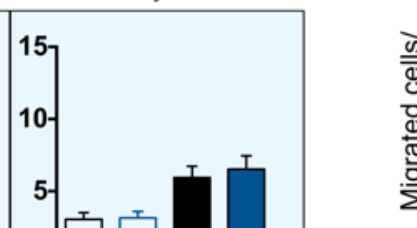

H
Ly6G+ neutrophil migration

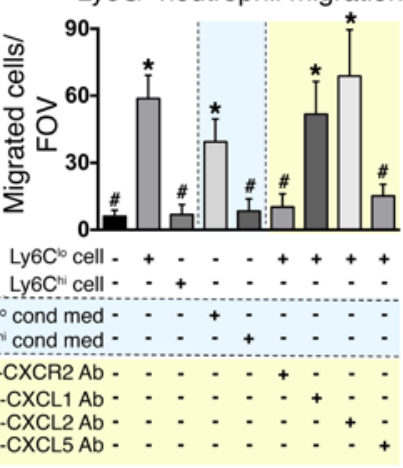

Figure 4. Ly6 ${ }^{10}$ monocyte infiltration during anti-VEGFR2 treatment recruits neutrophils via CXCL5. (A-D) Representative flow cytometry plots depicting subset-specific depletion of myeloid cells in (A) WT control, (B) Cx3cr7 $7^{-/-}$(Ly6C ${ }^{10}$ monocyte), (C) Ccr2 $2^{-/-}$(Ly6C ${ }^{\text {hi }}$ monocyte), and (D) anti-Ly6C

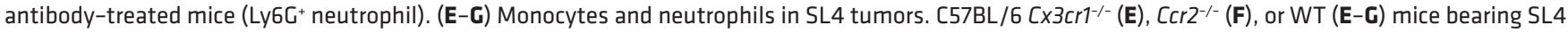
tumors were treated with either control rat IgG (C), anti-Ly6G antibody (C), DC101 (D), or anti-Ly6C antibody plus DC101 (G+D). Each subset of myeloid cells in tumor infiltrate was analyzed on day 12 by flow cytometry. $n=8$ /group. Comparison between groups was made using ANOVA with Holm-Šíák post-hoc test. ${ }^{*} P<0.05$. The graphs depict the absolute number of cells per $\mathrm{mg}$ of tumor tissue (E-G). Data are representative of 3 independent experiments. (H) In vitro migration assay. Neutrophils isolated from tumors were seeded in the upper chamber, and their migration to the bottom part of the chamber was measured. The lower chamber included either tumor-isolated $\mathrm{Ly}_{6 \mathrm{C}^{\mathrm{I}}}$ monocytes, Ly6C $\mathrm{Ch}^{\mathrm{hi}}$ monocytes, or their conditioned media with or without neutralizing antibodies for the chemokine/chemokine receptor as indicated. $n=9 /$ group. Comparison between groups was made using ANOVA with Holm-Šídák posthoc test. ${ }^{*} P<0.05$ versus control (first bar); ${ }^{\#} P<0.05$ versus Ly6 ${ }^{10}$ monocytes (second bar). Data are represented as mean $\pm S E M$. FOV, field of view.

showed reduced expression of CX3CL1 (Figure 3F). The reduction in CX3CL1 levels was recovered by using DC101 to block VEGF/VEGFR2 signaling (Figure 3F). These results suggest that CX3CL1 is produced by endothelial cells and that production is regulated by VEGFR2 signaling. Furthermore, since the elevation of CX3CL1 expression precedes the induction of hypoxia (Figure $3 \mathrm{E}$ and Supplemental Figure 1D), this process may not be hypoxia dependent, although we cannot rule out the contribution of hypoxia in the later time points. Thus, blockade of VEGF/VEGFR2 signaling stimulates robust upregulation of CX3CL1 and causes active recruitment of $\mathrm{CX} 3 \mathrm{CR} 1^{+} \mathrm{Ly} 6 \mathrm{C}^{\mathrm{lo}}$ monocytes to tumors.

Ly6C $C^{l o}$ monocyte infiltration during anti-VEGFR2 treatment recruits neutrophils. Based on these results, we hypothesized that DC101 treatment facilitates early infiltration of Ly6 $6 \mathrm{C}^{\mathrm{lo}}$ monocytes (day 5), which subsequently recruit neutrophils to these tumors (day 12) (Figure 1). To test this hypothesis, we selectively inhibited the infiltration of each myeloid cell subset by taking advantage of the unique expression of specific chemokine receptors on their surface, which are critical for their migration (i.e., CX3CR1 on Ly6 $\mathrm{C}^{\text {lo }}$ monocytes, CCR2 on Ly6 $\mathrm{C}^{\text {hi }}$ monocytes) $(32,59)$ (Supplemental Figure 2B). There was nearly $80 \%$ depletion of Ly $6 \mathrm{C}^{\text {lo }}$ monocytes in SL4 tumors growing in $\mathrm{Cx}_{3} \mathrm{cr}^{-/-}$mice when compared with WT mice (Figure 4, A, B, and E). In $\mathrm{Ccr}^{-/}$mice, there was an approximately $90 \%$ depletion of Ly $6 \mathrm{C}^{\text {hi }}$ monocytes (Figure 4, C and F). Finally, we used an anti-Ly6G neutralizing antibody to pharmacologically deplete the Ly6 $\mathrm{G}^{+}$neutrophils (with a depletion efficiency of $~ 80 \%$ ) (Figure 4, D and G). Interestingly, DC101-treated tumors in $\mathrm{C} \times 3 \mathrm{cr}^{-1-}$ mice showed not only a lack of Ly $6 \mathrm{C}^{\text {lo }}$ monocytes, but also significantly reduced infiltration of neutrophils compared with WT animals (Figure 4E). On the other hand, administration of an anti-Ly6G antibody selectively depleted $\mathrm{Ly}_{6} 6 \mathrm{G}^{+}$neutrophils without affecting Ly $6 \mathrm{C}^{\mathrm{lo}}$ monocytes (Figure $4 \mathrm{G}$ and Supplemental Figure 3B). These data indicate that early infiltration of Ly $6 \mathrm{C}^{\text {lo }}$ monocytes during anti-VEGFR2 treatment promotes subsequent recruitment of neutrophils to tumors.

Ly6C $C^{l o}$ monocytes attract neutrophils via CXCL5 during anti-VEGFR2 treatment in CRCs. We then investigated the underlying mechanisms of how Ly6 $\mathrm{C}^{\text {lo }}$ monocytes attract neutrophils. Ly6 $\mathrm{C}^{\text {lo }}$ monocytes grown in vitro significantly increased the number of neutrophils that migrated to the bottom part of a Boyden chamber, while Ly6 $\mathrm{C}^{\text {hi }}$ monocytes did not (Figure $4 \mathrm{H}$ ). DC101-treated tumors, characterized by abundant infiltration 
A
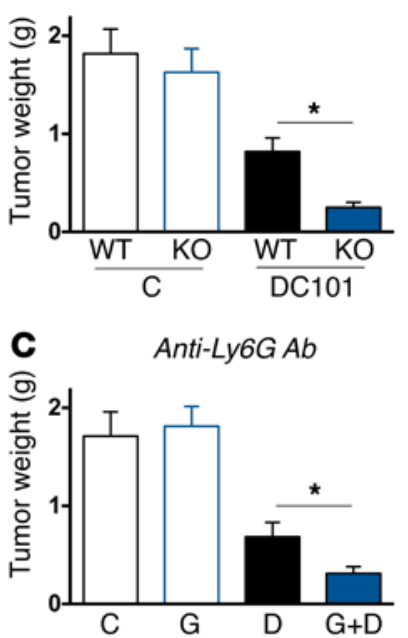

B $\quad \mathrm{Crr}^{-1}$
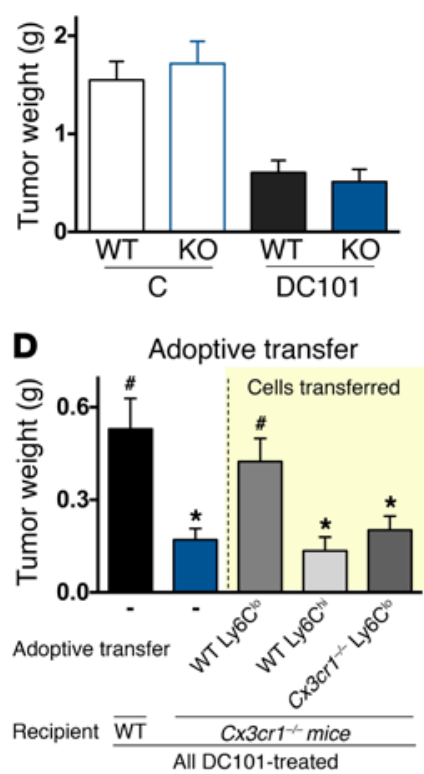

of Ly6 $\mathrm{C}^{\mathrm{lo}}$ monocytes compared with the control (Figure 1, D-G), had a significantly higher level of CXCL5, a chemokine known to attract CXCR2 ${ }^{+}$cells (Supplemental Figure 2D). Ly6C ${ }^{\text {lo }}$ monocytes secreted high levels of CXCL5 compared with Ly6 $\mathrm{C}^{\text {hi }}$ monocytes (Supplemental Figure 2, B and C). Since neutrophils expressed CXCR2 on their surface (Supplemental Figure 2B), we hypothesized that CXCL5 from Ly6 $\mathrm{C}^{\text {lo }}$ monocytes is a main chemoattractant for neutrophil recruitment. To verify this, we used anti-CXCR2 and anti-CXCL5 neutralizing antibodies and measured impaired neutrophil migration toward Ly6 $\mathrm{C}^{\text {lo }}$ monocytes (Figure $4 \mathrm{H}$ ). Other chemokines known to bind to CXCR2 (e.g., CXCL1 and CXCL2) did not seem to be crucial for attracting neutrophils in our models (Figure $4 \mathrm{H}$ ). These results support our hypothesis that Ly6C ${ }^{\text {lo }}$ monocytes secrete CXCL5 to recruit neutrophils expressing CXCR2.

Blockade of CX3CR1-dependent infiltration of Ly6C ${ }^{\text {lo }}$ monocytes improves efficacy of anti-VEGFR2 therapy. We next determined the in vivo function of each myeloid cell subset by utilizing the aforementioned strategies to specifically inhibit their infiltration to tumors (Figure 4). In $\mathrm{C} x 3 \mathrm{cr}^{1 /--}$ mice, which have reduced tumor infiltration of Ly6 $\mathrm{C}^{\text {lo }}$ monocytes and neutrophils (Figure 4E), DC101 monotherapy exerted an enhanced antitumor effect compared with the same treatment in WT mice (Figure 5A and Supplemental Figure 6A). On the other hand, depletion of Ly6 $\mathrm{C}^{\text {hi }}$ monocytes in $\mathrm{Ccr}^{-/-}$mice did not change the treatment efficacy of DC101 compared with WT mice (Figure 5B and Supplemental Figure 6B). Administration of an anti-Ly6G antibody, causing a significant reduction in $\mathrm{Ly} 6 \mathrm{G}^{+}$neutrophils, enhanced the antitumor effect of DC101, independent of monocyte infiltration (Figure 5C and Supplemental Figure 6C). Taken together, we conclude that the hindrance of either DC101-induced early infiltration of Ly6 $\mathrm{C}^{\text {lo }}$ monocytes or subsequent recruitment of neutrophils is sufficient to improve the antitumor efficacy of anti-VEGFR2 therapy.

Adoptive transfer of Ly6C lo monocytes abrogates improved efficacy of anti-VEGFR2 therapy in $\mathrm{Cx} 3 \mathrm{crl}^{-1-}$ mice. We next carried out

Figure 5. Blockade of CX3CR1-dependent infiltration of Ly6C ${ }^{10}$ monocytes improves efficacy of anti-VEGFR2 therapy. (A) SL4 tumors were grown

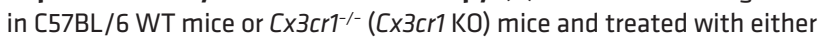
control rat IgC (C) or DC101. Tumor weight was measured on day 12 after treatment (A-D). (B) SL4 tumors were grown in C57BL/6 WT mice or $\mathrm{CCr}^{-1}$ (CCR2 KO) mice and treated as indicated. (C) SL4 tumor-bearing C57BL/6 WT mice were treated with either control rat IgG (C), anti-Ly6C antibody (C), DC101 (D), or anti-Ly6C antibody plus DC101 (G+D). Data are represented as mean \pm SEM. $n=8$ /group. Comparison between groups was made using ANOVA with Holm-Šídák post-hoc test. ${ }^{*} P<0.05$. Data are representative of 3 independent experiments (A-C). (D) DC101-treated $C \times 3 \mathrm{Cr}^{-1-}$ mice received adoptive transfer of either tumor-isolated WT Ly6 $\mathrm{C}^{\mathrm{C}}$ monocytes (Ly6C $\left.{ }^{10}\right)$, WT Ly6C ${ }^{\text {hi }}$ monocytes (Ly6C $\left.C^{\text {hi }}\right)$, or Ly6 ${ }^{10}$ monocytes isolated from tumors of $\mathrm{C}_{3} \mathrm{Cr}^{-1-}$ mice (KO Ly6C ${ }^{\circ}$ ) twice a week from the beginning of DC101 treatment. Data are represented as mean \pm SEM. $n=8 /$ group. Comparison between groups was made using ANOVA with Holm-Šídák post-hoc test. ${ }^{*} P<0.05$ versus without cell transfer (black bar); ${ }^{\#} P<0.05$ versus $\mathrm{C} \times 3 \mathrm{CrT}^{-/-}$control mice without cell transfer (blue bar).

a series of adoptive transfer experiments to test whether Ly6 $\mathrm{C}^{\mathrm{lo}}$ monocytes could "rescue" the phenotype in $\mathrm{C} x 3 \mathrm{CrI}^{-/}$mice. Tumor weight of DC101-treated $\mathrm{Cx} 3 \mathrm{Cr}^{-/-}$mice that received adoptive transfer of WT Ly $6 \mathrm{C}^{\text {lo }}$ monocytes was significantly higher than that in DC101-treated $\mathrm{Cx}_{3} \mathrm{cr}^{1 /-}$ mice without cell transfer (Figure 5D). We confirmed that the adoptive transfer increased the numbers of Ly $6 \mathrm{C}^{\text {lo }}$ monocytes and neutrophils in the tumors in $\mathrm{C} x 3 \mathrm{crI}^{-/-}$mice (Supplemental Figure 6, D-F). We also adoptively transferred Ly6C $\mathrm{C}^{\mathrm{lo}}$ monocytes isolated from $\mathrm{Cx} 3 \mathrm{crl}^{-/-}$mice. Since Ly6C $\mathrm{C}^{\mathrm{lo}}$ monocytes from $\mathrm{Cx} 3 \mathrm{cr}^{1 /-}$ mice lack CX3CR1 expression on their surface, these adoptively transferred cells did not infiltrate into tumors and tumor weights were not increased (Figure 5D and Supplemental Figure 6D). Also, adoptive transfer of Ly6 $\mathrm{C}^{\text {hi }}$ monocytes did not increase the tumor weight compared with the DC101-treated $\mathrm{C} x 3 \mathrm{crr}^{-/-}$mice without cell transfer (Figure 5D). These data suggest that CX3CR1 signaling is the key mechanism driving Ly $6 \mathrm{C}^{\text {lo }}$ monocyte infiltration in CRCs and that Ly $6 \mathrm{C}^{\mathrm{lo}}$ monocytes influence tumor growth.

Ly6C $C^{l o}$ monocytes drive immunosuppression during anti-VEGFR2 treatment in CRCs. We next asked how antiangiogenic therapyinduced Ly6 $\mathrm{C}^{\text {lo }}$ monocyte infiltration supports tumor growth. As shown in Supplemental Figure 1C, we observed vessel regression on days 5 and 12 after DC101 treatment without any rebound of vessel density, suggesting that tumor-infiltrating Ly6 $\mathrm{C}^{\text {lo }}$ monocytes or neutrophils do not seem to promote tumor angiogenesis. Consistent with this, we did not observe any significant difference in levels of Bv8 expression - previously implicated in the antiangiogenesis therapy resistance process by promoting angiogenesis (23) - between DC101-treated and control tumors in our models (Supplemental Figure 1, E and F).

We next asked whether the tumor-infiltrated Ly6C $\mathrm{C}^{\text {lo }}$ monocytes are able to modulate the tumor immune microenvironment. We found that expression levels of immunosuppressive cytokines (i.e., IL-10 and TGF- $\beta 1$ ) were high in both Ly6 $\mathrm{C}^{\text {lo }}$ monocytes and neutrophils in vitro (Supplemental Figure 2, B and C). In vivo, we measured higher levels of immunosuppressive cytokines in DC101-treated tumors, which are abundantly infiltrated by Ly6 $\mathrm{C}^{\text {lo }}$ monocytes and neutrophils, than in the control. Further, immunostimulatory cytokines (i.e., TNF- $\alpha$ and IL-2) were downregulated upon DC101 treatment (Figure 6A and Supplemental Figure 2D). Flow cytometry analyses showed that DC101-treated tumors have 
A

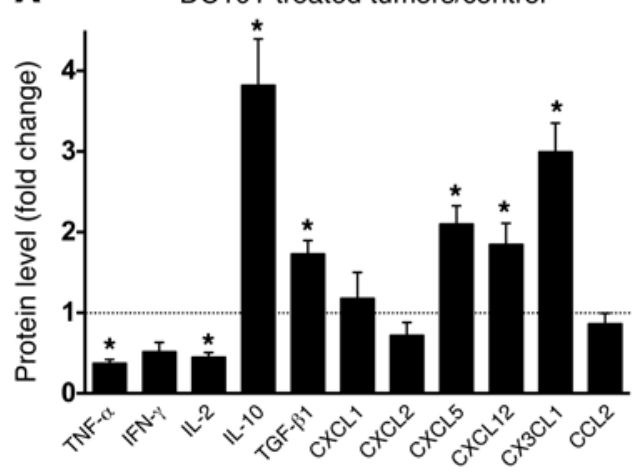

B

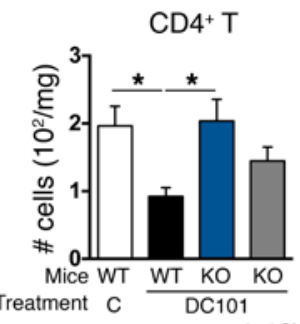

Adoptive transfer - - - Ly6C
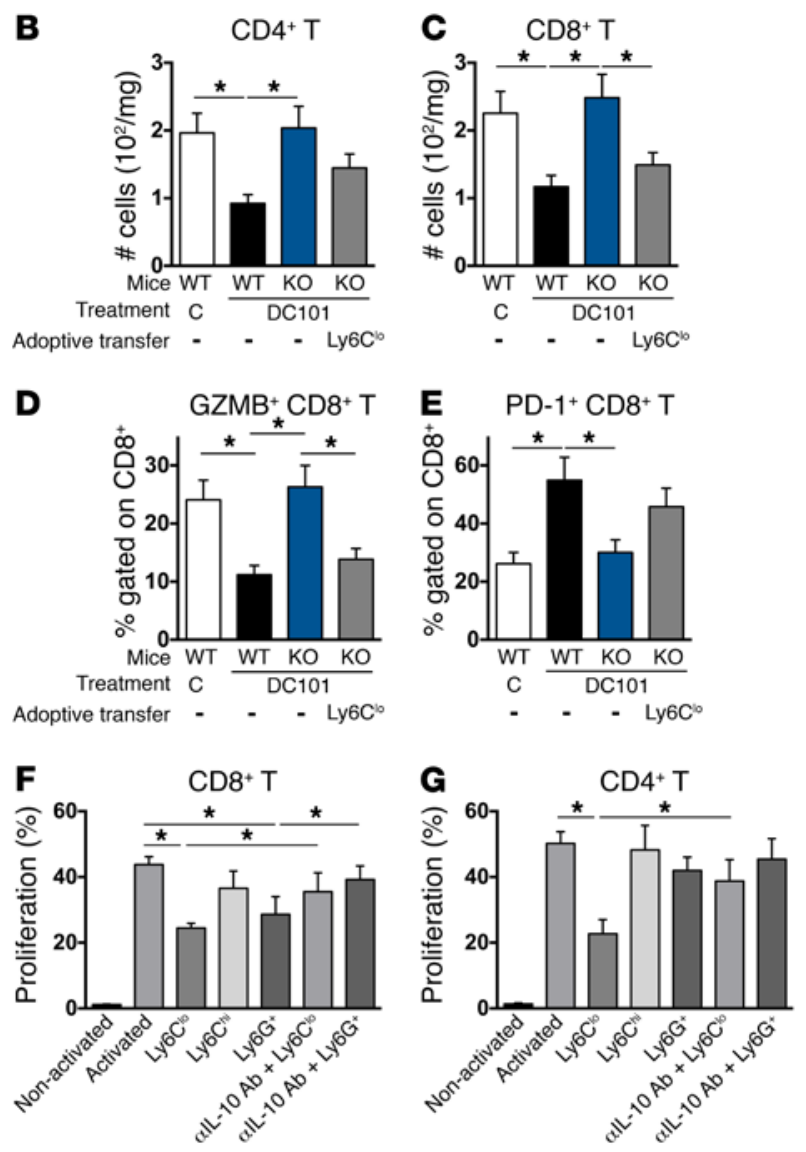

significantly fewer effector $\mathrm{CD} 4^{+}$and $\mathrm{CD} 8^{+} \mathrm{T}$ cells compared with control (Figure 6, B and C). Interestingly, lymphocytes in DC101-treated tumors expressed more PD-1, while lymphocytes in control-treated tumors showed more granzyme B expression (Figure 6, D and E, and Supplemental Figure 7A). Furthermore, tumors from DC101-treated $\mathrm{Cx} 3 \mathrm{crr}^{-/-}$mice, which showed delayed tumor growth (Figure $5 \mathrm{~A}$ ), had higher numbers of $\mathrm{CD}^{+}$and $\mathrm{CD} 8^{+}$ $T$ cells, with more granzyme $\mathrm{B}$ and less $\mathrm{PD}-1$ expression, compared with DC101-treated WT animals (Figure 6, B-E, and Supplemental Figure 7A). Importantly, the higher numbers of $\mathrm{CD}^{+}$and $\mathrm{CD}^{+}$ $\mathrm{T}$ cells in $\mathrm{C} \times 3 \mathrm{crr}^{-1-}$ mice were ablated when we adoptively transferred WT Ly6 $\mathrm{C}^{\mathrm{lo}}$ monocytes into $\mathrm{Cx} 3 \mathrm{crr}^{-/-}$mice (Figure 6, B-E). These data strongly support the mechanism that Ly $6 \mathrm{C}^{\mathrm{lo}}$ monocytes are directly involved in the regulation of adaptive immunity
Figure 6. Ly6 $\mathrm{C}^{10}$ monocytes drive immunosuppression during antiVEGFR2 treatment in CRCs. (A) C57BL/6 WT mice bearing syngeneic orthotopic SL4 tumors were treated with either control rat IgG or DC101. Protein levels were measured on day 12 after treatment from tumor tissue lysates (Supplemental Figure 2D). (B and C) Flow cytometric analysis of $\mathrm{CD}^{+}$(B) and CD8 ${ }^{+}$T cells (C) in SL4 tumors as indicated. White bar, WT mice bearing SL4 tumors treated with control rat lgG; black bar, WT mice bearing SL4 tumors treated with DC101; blue bar, $\left[\times 3 \mathrm{Cr}^{-1-}\right.$ mice bearing SL4 tumors treated with DC101 without cell transfer; gray bar, DC101-treated $\mathrm{C}_{3} \mathrm{Cr}^{-1-}$ mice received adoptive transfer of tumor-isolated WT Ly6C ${ }^{10}$ monocytes. The graphs depict data for the absolute number of cells per mg of tumor tissue (B and $\mathbf{C}$ ). The lymphocyte infiltrate in the tumor was analyzed on day 12 by flow cytometry. ( $\mathbf{D}$ and $\mathbf{E}$ ) Flow cytometric analysis of $\mathrm{CD}^{+} \mathrm{T}$ cells. The graphs depict data for granzyme $\mathrm{B}^{+}$ (D) or PD-1+ (E) populations relative to total CD8 ${ }^{+} \mathrm{T}$ cells. The lymphocyte infiltrate in the tumor was analyzed on day 12 by flow cytometry. $n=8$ / group. Data are represented as mean \pm SEM. ${ }^{*} P<0.05$. (F and $\left.\mathbf{C}\right)$ CFSEbased $T$ cell proliferation assays. CellTrace-labeled splenic CD8 ${ }^{+}(\mathbf{F})$ or CD4 ${ }^{+} T$ cells (C) from syngeneic mice were activated and coincubated with either tumor-isolated Ly6C ${ }^{10}$ monocytes, Ly6C ${ }^{\text {hi }}$ monocytes, or neutrophils with or without anti-IL-10 neutralizing antibody as indicated. $n=3 /$ group Data are represented as mean \pm SEM. (B-C) Comparison between groups was made using ANOVA with Holm-Šídák post-hoc test. ${ }^{*} P<0.05$. Data are representative of $\mathrm{B}$ independent experiments.

and suggest that DC101-treated tumors became skewed toward an immunosuppressive phenotype by infiltration of Ly6 $\mathrm{C}^{\mathrm{lo}}$ monocytes (Figure 6, A-E, and Supplemental Figure 7).

Ly6C ${ }^{l o}$ monocytes and neutrophils produce IL-1O and inhibit $T$ lymphocyte proliferation. Since Ly $6 \mathrm{C}^{\mathrm{lo}}$ monocytes and neutrophils were more abundant in tumors with an immunosuppressive microenvironment, we further evaluated their capacity to suppress the proliferation of activated $\mathrm{T}$ lymphocytes. An in vitro CFSE assay revealed that both Ly6 $\mathrm{C}^{\text {lo }}$ monocytes and neutrophils inhibited $\mathrm{CD}^{+} \mathrm{T}$ cell proliferation (Figure $6 \mathrm{~F}$ ). Ly6 $\mathrm{C}^{\text {lo }}$ monocytes also prevented the proliferation of $\mathrm{CD}^{+} \mathrm{T}$ lymphocytes (Figure 6G). Furthermore, motivated by the findings that Ly6 $\mathrm{C}^{\text {lo }}$ monocytes and neutrophils express a high amount of IL-10 (Supplemental Figure 2, B and C), we found that treatment with an anti-IL-10 neutralizing antibody prevented Ly6 $\mathrm{C}^{\mathrm{lo}}$ monocytes and neutrophils from inhibiting $\mathrm{T}$ cell proliferation (Figure 6, F and G). Thus, we hypothesize that DC101-induced recruitment of Ly $6 \mathrm{C}^{\text {lo }}$ monocytes and neutrophils producing IL-10 inhibits effector $\mathrm{T}$ cell activation, leading to a shift of the tumor microenvironment toward immunosuppression and thus to an attenuated immune response against the tumor.

In vivo nanoparticle delivery of siCX $3 C L 1$ inhibits $L y 6 C^{l o}$ monocyte infiltration and enhances efficacy of anti-VEGFR2 therapy. To determine whether CX3CL1 is an initiating molecule to DC101 tumor resistance, we developed a gene therapy method that can be potentially translated into an effective adjunct to anti-VEGF therapy in the clinic using nanoparticles $(7 \mathrm{C} 1)$ delivering siRNA to target endothelial cells in vivo (60) (Figure 7A).

To validate whether the application of 7C1 nanoparticles was feasible for targeting endothelial cells in the tumor microenvironment, we first used nanoparticles formulated with siTIE2, which has already been proven to be efficacious in silencing Tie2 mRNA in several tissues (60). There was a significant decrease in TIE2 expression levels after 7C1-siTIE2 treatment in CRCs (Supplemental Figure 8A). Next, we needed to harness specific siRNA 
A

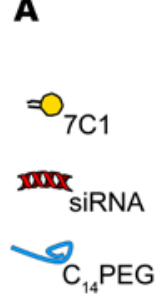

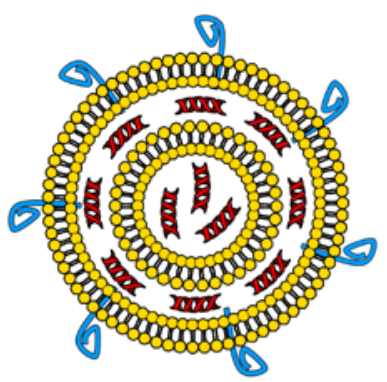

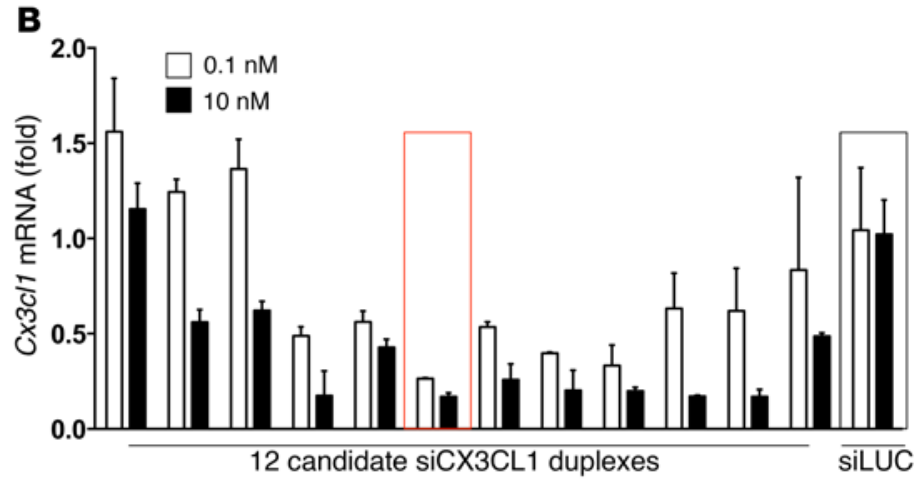

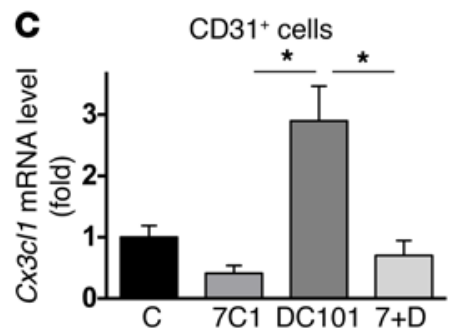
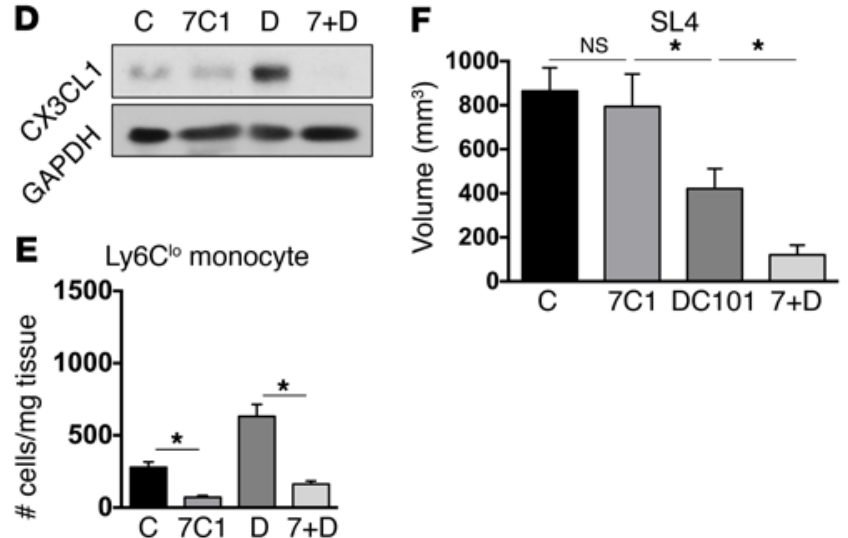

Figure 7. In vivo nanoparticle delivery of siCX3CL1 inhibits Ly6C monocyte infiltration and enhances efficacy of anti-VECFR2 therapy. (A) Schematic of 7C1 nanoparticle formulated with siRNA. (B) In vitro screening of siCX3CL1 candidate duplexes. Relative CX3c/1 expression level normalized to siLUC (luciferase) control is plotted for candidate duplexes in $0.1 \mathrm{nM}$ or $10 \mathrm{nM}$. Each siRNA was transfected twice, and mRNA analysis was run in triplicate. Red box indicates the best duplex selected for large-scale synthesis and subsequent nanoparticle formulation. Black box indicates siRNA control that targets luciferase. (C-F) C57BL/6 WT mice bearing orthotopically grown syngeneic SL4 CRCs were treated with either control rat IgC (C), 7C1-Axo-siCX3CL1 (7C1), DC101 (D), or 7C1-Axo-siCX3CL1 plus DC101 (7+D). (C) Relative Cx3c/1 mRNA expression levels in endothelial cells isolated from SL4 tumors were determined by quantitative real-time PCR, normalized against Gapdh. Data are represented as mean \pm SEM. $n=8 /$ group. Comparison between groups was made using ANOVA with Holm-Šídák post-hoc test. ${ }^{*} P<0.05$. (D) Western blot analysis of CX3CL1 protein expression in SL4 tumors treated as indicated. CX3CL1 protein levels were measured on day 12 after treatment. (E) Ly6 $6 C^{10}$ monocytes in SL4 tumors treated as indicated. Ly6 $6 C^{10}$ monocytes in tumor infiltrate were analyzed on day 12 after treatment by flow cytometry. $n=8$ /group. The graphs depict the absolute number of cells per mg of tumor tissue. (F) Tumor volume of SL4 measured on day 12 after treatment. $n=8$ /group. Data are represented as mean \pm SEM. Comparison between groups was made using ANOVA with Holm-Šídák post-hoc test. ${ }^{*} P<0.05$.

sequences with superior knockdown efficacy against CX3CL1 (siCX3CL1), especially when applied in vivo. We performed in vitro screening with 12 candidate sequences, identified as lead siRNA molecules by in silico predictions of target specificity and activity (Figure 7B). The best duplex with sequence 5'-gcuuGcGAGAGGGuuuAAAdTsdT-3' (sense) (where upper-case letters represent unmodified RNA [2'-OH] residues and lower-case letters represent residues with 2'-O-methyl modification) and 5'- UUuAAACCCUCUCGcAAGCdTsdT-3' (anti-sense) was selected for largescale synthesis and subsequent nanoparticle formulation (Figure 7B and Supplemental Figure 8B). Importantly, when we compared the knockdown efficiency of our siCX3CL1 (hereafter referred to as Axo-siCX3CL1) and another siRNA against CX3CL1 from a recent publication (61), there was a dramatic enhancement in silencing efficiency for Axo-siCX3CL1 (Supplemental Figure 8C).

Next, we examined the effect of 7C1-Axo-siCX3CL1 in combination with DC101 in CRCs. Treatment with the negative control 7C1-siLUC (silencing luciferase mRNA) did not change tumor growth or immune cell infiltration, and there was no difference between the DC101 group and 7C1-siLUC plus DC101 group (Supplemental Figure 8, D and E). We found that treating with 7C1-Axo-siCX3CL1 significantly enhanced the antitumor effect of anti-VEGFR2 therapy, even though there was negligible effect with 7C1-Axo-siCX3CL1 alone (Figure 7F and Supplemental Figure 8F). We confirmed that treatment of 7C1-Axo-siCX3CL1 markedly reduced DC101-induced upregulation of CX3CL1 in endothelial cells by measuring both mRNA and protein levels (Figure 7, C and D). We also observed that in vivo knockdown of $C x 3 c l 1$ mRNA significantly inhibited the infiltration of Ly $6 \mathrm{C}^{\text {lo }}$ monocytes into DC101-treated tumors and that subsequent tumor growth was delayed compared with control with only DC101 treatment (Figure 7, E and F). Consistent with our experiments using $\mathrm{C} \times 3 \mathrm{crr}^{-/-}$mice, 7C1-Axo-siCX3CL1treated tumors also showed subsequent decrease in $\mathrm{Ly}_{6} \mathrm{G}^{+}$neutrophils, but did not alter Ly6 $\mathrm{C}^{\text {hi }}$ monocytes (Figure $4 \mathrm{E}$ and Supplemental Figure 8, G and H). These data confirm that CX3CL1 is an important chemoattractant for Ly6 $\mathrm{C}^{\mathrm{lo}}$ monocytes and contributes to the process of anti-VEGF therapy resistance. 


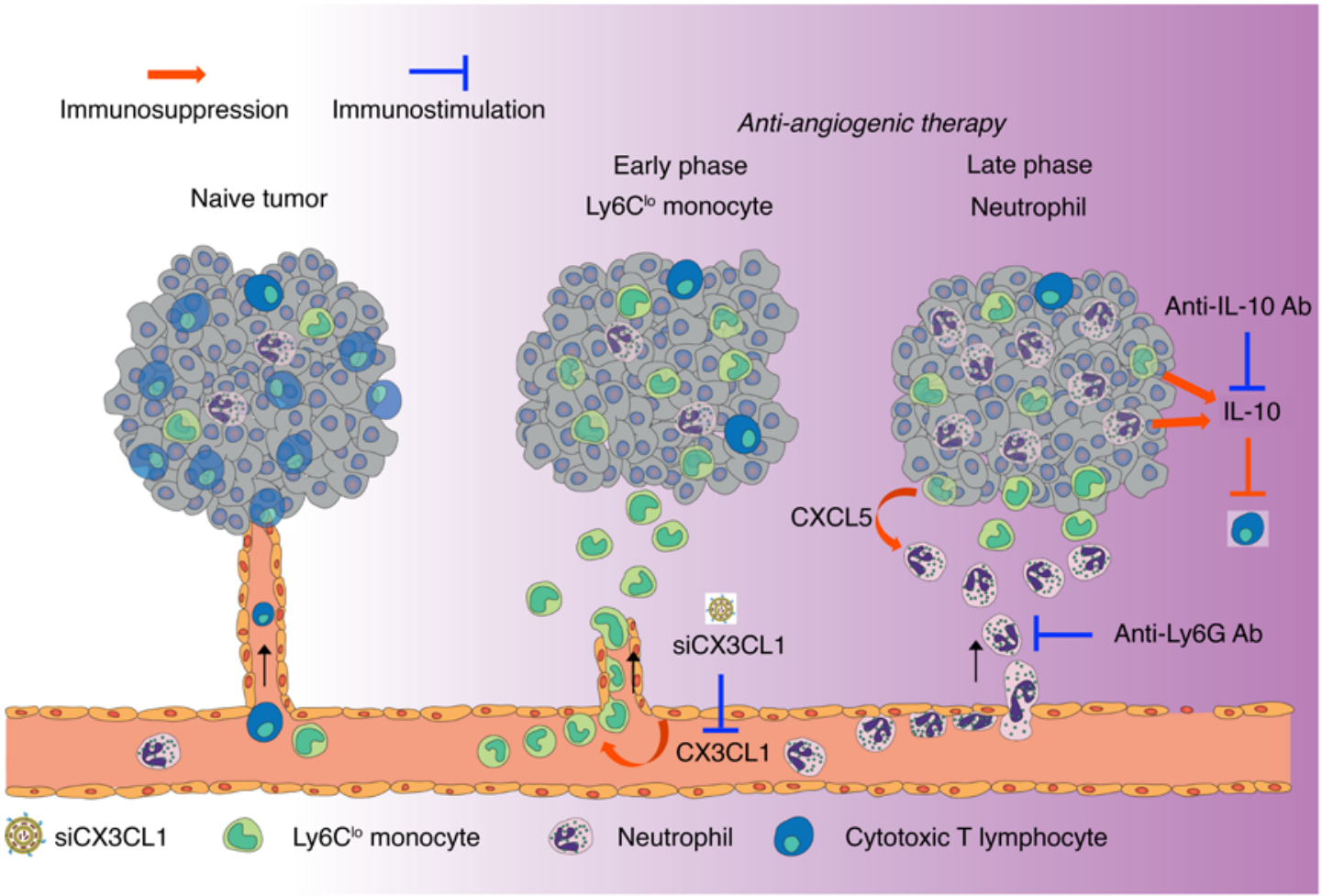

Figure 8. Proposed mechanism of antiangiogenic therapy-induced immunosuppression. Anti-VEGFR2 therapy upregulates the expression of CX3CL1 that recruits CX3CR $1^{+}{ }^{+} \mathrm{Ly} 6 \mathrm{C}^{\mathrm{Io}}$ monocytes (center, early phase), which subsequently attracts neutrophils via CXCL5 (right, late phase), resulting in the formation of an immunosuppressive microenvironment with a reduction of cytotoxic $T$ lymphocytes in the tumor. The multistep process provides multiple points of intervention to prevent immune resistance and improve the effectiveness of anti-VEGF therapy. Red arrows indicate steps in the immunosuppressive cascade, which can be targeted as demonstrated in this study (blue inhibition arrow).

\section{Discussion}

Here we report, for what we believe is the first time, the immunosuppressive functions of Ly $6 \mathrm{C}^{\text {lo }}$ monocytes and identify them as an important driver of resistance during anti-VEGFR2 treatment in CRCs. To date, studies have divided $\mathrm{Gr}^{+}$myeloid-derived suppressor cells (MDSCs) into 2 different subpopulations, monocytic and granulocytic (also known as polymorphonuclear) MDSCs (25-27, 42, 62). However, Gr1 is not a single surface marker, but rather a complex of proteins Ly6C and Ly6G. Due to the complexity of Gr1, previous studies that utilized Gr1 staining were not able to provide a clear separation of the subpopulations (62). Recent reports that adopted Ly6C and Ly6G for subpopulation separation focused only on $\mathrm{Gr}^{\text {hi }}{ }^{\text {hi }}$ myeloid cells, which include $\mathrm{Gr}^{+}\left(\mathrm{Ly} 6 \mathrm{C}^{\mathrm{hi}}\right)$ monocytes and granulocytes (39). Moreover, the definition of the myeloid cell subpopulations using surface markers has been ambiguous among research groups $(35-38,42,43)$. Unlike $\mathrm{Gr}^{+}$ monocytes and granulocytic cells $(6,23,24)$, Ly6 $\mathrm{C}^{\text {lo }}$ monocytes represent a distinct cell population that has never been studied for its role in conferring resistance to anti-VEGF therapy.

As described above, there have been reports on the presence of several myeloid cells (i.e., $\mathrm{Gr} 1^{+}, \mathrm{Ly} 6 \mathrm{C}^{\text {hi }}$, or TIE2 ${ }^{+}$monocytes and granulocytic cells) and their respective roles in resistance to antiangiogenic cancer therapy. However, our data show that Ly6 $\mathrm{C}^{\text {lo }}$ monocytes along with their immunosuppressive functions form a distinct population of myeloid cells, which are immunophenotypically different from the $\mathrm{Gr}^{+}$or $\mathrm{TIE} 2^{+}$monocytes and granulocytic cells described previously. We identified Ly6 $\mathrm{C}^{\text {lo }}$ monocyte infiltration after anti-VEGFR2 therapy, and these cells were not observed in previous reports on anti-VEGF cancer therapy. Our study is the first report, to our knowledge, that investigates the ability of Ly $6 \mathrm{C}^{\text {lo }}$ monocytes to confer resistance to anti-VEGF cancer therapy.
Furthermore, we find that immunosuppression, rather than angiogenesis, in the tumor microenvironment is the key mechanism conferring resistance to anti-VEGF therapy exerted by Ly $6 \mathrm{C}^{\text {lo }}$ monocytes. Previous reports implicated proangiogenic functions of myeloid cells or monocytes (i.e., $\mathrm{CD} 11 \mathrm{~b}^{+} \mathrm{Gr} 1^{+}$cells or $\mathrm{Ly}^{6} \mathrm{G}^{+}$ granulocytes) in anti-VEGF therapy resistance in some tumors, not their immune-regulatory functions.

Some reports show that low doses of anti-VEGF therapy can induce vascular normalization and improve antitumor immunity $(63,64)$. It is also known that high dose or prolonged treatment of anti-VEGF therapy promotes hypoxia and immunosuppression in the tumor microenvironment in both clinical and preclinical studies $(1,6,24,65-68)$. The latter case explains one mechanism of anti-VEGF therapy resistance in patients, which is consistent with our observations in CRC models. Indeed, the therapeutic dose of bevacizumab currently used in the clinic is often considered as a high dose (69), which is comparable to the dose we used in our study (maximum effective dose). These findings imply that immune resistance may hinder responsiveness to anti-VEGF/ VEGFR therapy. Here, we claim that high-dose anti-VEGFR2 therapy induces immunosuppression and that this occurs via an endothelial CX3CL1/Ly6 $\mathrm{C}^{\mathrm{lo}} \mathrm{CX} 3 \mathrm{CR} 1^{+}$monocyte mechanism.

In this study, we clearly distinguished 3 different innate immune cell subpopulations based on their immunophenotype (i.e., Ly6C and Ly6G) (Figure 1C). Although Ly6C ${ }^{\text {lo }}$ monocytes have been described and characterized in previous publications, studies on their roles in vivo have been mostly in noncancer settings $(32,48,49)$. Interestingly, Hanna et al. recently reported that patrolling Ly $6 \mathrm{C}^{\mathrm{lo}}$ monocytes are important in recruiting NK cells to prevent cancer metastasis in the lung, which is characterized by an exceptionally abundant NK cell population compared with other 
tissues (70). However, the immunosuppressive functions of Ly $6 \mathrm{C}^{\text {lo }}$ monocytes have not been reported in any context, especially in primary tumors during the process of antiangiogenic therapy resistance. Of note, we observed only a negligible number of NK cells in our CRC models (Supplemental Figure 7A), similar to other tumor models available in our laboratory.

In our CRC models, the expression levels of immunosuppressive cytokines (i.e., IL-10 and TGF- $\beta 1$ ) were high in both Ly6C ${ }^{\text {lo }}$ monocytes and neutrophils. DC101-treated tumors, abundantly infiltrated by Ly6 $\mathrm{C}^{\text {lo }}$ monocytes and neutrophils, were composed of significantly $(P<0.05)$ fewer effector $\mathrm{CD} 4^{+}$and $\mathrm{CD} 8^{+} \mathrm{T}$ cells, and those $\mathrm{T}$ cells that were present expressed more PD-1 and less granzyme B. This phenotype was ablated in $\mathrm{Cx}_{3} \mathrm{Cr}^{-1-}$ mice. An in vitro CFSE assay revealed that $\mathrm{Ly}_{6 \mathrm{C}^{\mathrm{lo}}}$ monocytes inhibited $\mathrm{CD} 4^{+}$ and $\mathrm{CD}^{+} \mathrm{T}$ cell proliferation, a phenotype reversed by using an anti-IL-10 neutralizing antibody (Figure 6, F and G). Thus, DC101induced recruitment of IL-10-producing Ly $6 \mathrm{C}^{\text {lo }}$ monocytes and neutrophils shifted the tumor microenvironment toward immunosuppression, leading to less infiltration of cytotoxic effector T lymphocytes. Recently, our group showed that modulation of innate immune cells (i.e., tumor-associated macrophages[TAMs]) subsequently regulates the activity of cytotoxic $\mathrm{T}$ cells in breast cancer models and that depletion of the cytotoxic T cells using anti-CD8 neutralizing antibody abrogated the effect of TAM modulation (63). Therefore, if we deplete $\mathrm{CD}^{+} \mathrm{T}$ cells in our colon cancer model after blocking Ly6 $\mathrm{C}^{\mathrm{lo}}$ monocyte infiltration, we would expect abrogated antitumor immunity even with decreased numbers of Ly6 $\mathrm{C}^{\text {lo }}$ monocytes in tumors.

By genetically or pharmacologically depleting one specific subset of myeloid cells at a time, we found that Ly6 $\mathrm{C}^{\text {lo }}$ monocyte infiltration promoted subsequent neutrophil recruitment during anti-VEGFR2 treatment (Figure 4). We also confirmed that the adoptive transfer of Ly6 $\mathrm{C}^{\text {lo }}$ monocytes alone increased the numbers of both $\mathrm{Ly} 6 \mathrm{C}^{\mathrm{lo}}$ monocytes and neutrophils in tumors of $\mathrm{C} \times 3 \mathrm{cr}^{1 /-}$ mice. Furthermore, these early infiltrating Ly6C $\mathrm{C}^{\text {lo }}$ monocytes overexpressed the chemokine CXCL5, which attracted $\mathrm{CXCR}^{+}$neutrophils. Other chemokines known to bind to CXCR2 (i.e., CXCL1 and CXCL2) did not seem to be important in attracting neutrophils in our models (Figure 4H, Figure 6A, and Supplemental Figure 2D), even though CXCL1 was previously proposed as a neutrophil attractant secreted from $\mathrm{Ly} 6 \mathrm{C}^{\mathrm{lo}}$ monocytes in nontumor models (49).

Based on our findings, we sought to develop a therapeutic strategy with the potential for clinical translation. We hypothesized that therapeutic targeting of CX3CL1 would selectively and potently block the infiltration of Ly6 $\mathrm{C}^{\mathrm{lo}}$ monocytes and improve the efficacy of anti-VEGF/VEGFR2 cancer therapy. To specifically and effectively silence CX3CL1, we utilized a gene-therapy approach, taking advantage of the recent advances in siRNA design and chemistry that allow the identification of specific and highly potent sequences with minimal immune stimulation and maximal siRNA stability. We further benefited from the utilization of nanoparticle formulations capable of efficacious siRNA delivery to tumor endothelial cells with clinically suitable delivery materials (7C1).

Tumor growth was significantly $(P<0.05)$ delayed in combined 7C1-Axo-siCX3CL1 and DC101-treated mice compared with the DC101 single-treatment group. Based on the promising therapeutic benefits observed in this study, we look forward to further applications of 7C1 nanoparticles for treatment strategies of various diseases.

While it is clear that endothelial cells in the CRC microenvironment produce and upregulate CX3CL1 expression upon anti-VEGFR2 treatment, it is conceivable that there may be other cell types expressing CX3CL1 in the tumor microenvironment. Here, we demonstrate that targeting CX3CL1 in endothelial cells is sufficient to block the infiltration of $\mathrm{Ly}^{6} \mathrm{C}^{\text {lo }}$ monocytes and improve survival (Figure 7). These data indicate endothelial cellderived CX3CL1 plays a key functional role in the recruitment of Ly6C ${ }^{\text {lo }}$ monocytes in CRCs during anti-VEGFR2 treatment.

It could be argued that the recruitment of Ly6 $6 \mathrm{C}^{\text {lo }}$ monocytes might be explained by the antibody opsonization of the endothelial cells that express VEGFR2. However, we think this is not the case, as the increase in tumor infiltration of Ly $6 \mathrm{C}^{\text {lo }}$ monocytes was mimicked by the treatment of an antibody blocking VEGF (data not shown).

Tumors often escape antitumor immune responses through critical immune checkpoint molecules. The recent approval of drugs targeting PD-1 or CTLA-4 shows the potential for inhibiting these pathways. However, this strategy is effective only in some tumor types and in only a portion of patients. Recently, 2 studies revealed that inhibition of granulocyte recruitment into tumors improves the efficacy of the immune checkpoint blockade $(35,41)$. Our data describing the immunosuppressive functions of Ly $6 \mathrm{C}^{\text {lo }}$ monocytes identify another path for the development of therapeutic strategies that can create synergy with the FDA-approved immune checkpoint inhibitors.

In addition, our cecum-imaging window, developed in this study, enabled quantification of dynamic mobilization of Ly6 $\mathrm{C}^{\text {lo }}$ monocytes with various types of behaviors over time, unveiling their CX3CR1-dependent infiltration into the tumor from the blood. The cecum window allowed longitudinal imaging for over 4 weeks, unparalleled by other imaging windows for the gut, that are applicable only for acute or short-term monitoring. The cecum window can be more broadly applied for investigations of both malignant and nonmalignant chronic diseases of the gut, such as inflammatory bowel disease and disorders related to the gut microbiota.

In summary, we found that $\mathrm{Ly} 6 \mathrm{C}^{\mathrm{lo}}$ monocytes are important drivers of resistance to antiangiogenic therapy in CRCs through their immunosuppressive functions. Moreover, the increase in CX3CL1 after antiangiogenic therapy in mouse models mirrored the findings in human tumor specimens. This supports our model that CX3CL1 upregulation results in the recruitment of Ly6C $\mathrm{C}^{\text {lo }}$ monocytes, which attract neutrophils to the tumor via CXCL5 and inhibit effector $\mathrm{T}$ cell formation (Figure 8 ). The multistep process provides multiple points of intervention to prevent immune suppression and improve the effectiveness of anti-VEGF therapy by modulating the immune microenvironment.

\section{Methods}

For more details, see Supplemental Methods.

Animals. Cx3cr1 ${ }^{g f / g f p}$ mice were originally provided by Dan R. Littman (New York University School of Medicine, New York, New York, USA) (51). Ccr2 ${ }^{--}$mice were purchased from Jackson Laboratories (stock number 004999). Homozygous $C x 3 c r 1^{g f p / g f p}$ mice were used for the $C \times 3 c r 1$-deficient model. $C \times 3 c r 1^{g f p /+}$ mice were obtained by breeding 
$C x 3 c r 1^{g f p / s f p}$ mice with $\mathrm{C} 57 \mathrm{BL} / 6$ WT mice. $C x 3 \mathrm{cr} 1^{1 f p /+}$ mice have $1 \mathrm{Cx} 3 \mathrm{cr} 1$ allele replaced with cDNA encoding Egfp. Mice were 8 to 10 weeks old.

CRC cell preparation. SL4 (71) murine CRC cells were cultured in DMEM/F12 1:1 mixture medium supplemented with 10\% FBS, and CT26 (72) murine CRC cells were cultured in RPMI-1640 medium supplemented with $10 \%$ FBS prior to implantation. Subconfluent SL4 or CT26 cells were harvested, washed with PBS, and counted. Tumor cell suspension was mixed with Matrigel (catalog 354262, Corning) in a 1 to 1 proportion by volume.

Orthotopic CRC and spontaneous rectal tumor model and treatment regimen. For the orthotopic CRC model, 8- to 10-week-old male C57BL/6J (for SL4 implantation) and BALB/c (for CT26) mice were anesthetized with intraperitoneal injection of ketamine $(100 \mathrm{mg} / \mathrm{kg})$ and xylazine $(10 \mathrm{mg} / \mathrm{kg})$. Abdominal hair was removed, and a $10-\mathrm{mm}$ midline incision was made. The cecum was exteriorized, and $5 \times 10^{5}$ cells in $10 \mu \mathrm{l}$ of PBS/Matrigel complex were injected into the cecal wall between the serosa and mucosa from the serosal side using an insulin syringe with a 27 -gauge needle $(71,72)$. The cecum was returned to the abdominal cavity, and the abdominal wall was closed with 5-0 polysorb sutures (Covidien), followed by skin closure with surgical staples. Tumor size was monitored either by measuring the activity of secreted Gaussia luciferase (GLuc) $(73,74)$ or by imaging with ultrasound twice a week. For the spontaneous rectal tumor model, conditional Apc knockout mice were used as described previously (33).

When tumor diameter reached $4 \mathrm{~mm}$, tumor-bearing mice were randomly assigned into different treatment groups and treated accordingly. Every 3 days, $40 \mathrm{mg} / \mathrm{kg}$ of DC101 (ImClone Systems/Eli Lilly), a monoclonal anti-VEGFR2 antibody, was administrated intraperitoneally. Control mice received $40 \mathrm{mg} / \mathrm{kg}$ of rat IgG intraperitoneally every 3 days. To deplete neutrophils, $5 \mathrm{mg} / \mathrm{kg}$ of anti-Ly6G antibody (BioXCell, clone 1A8, catalog BE0075-1) was administered intraperitoneally every 3 days. To silence Tie 2 mRNA, $1 \mathrm{mg} / \mathrm{kg}$ of 7C1-siTIE 2 was injected intravenously. To silence Cx3cl1 mRNA, $1 \mathrm{mg} / \mathrm{kg}$ of 7C1Axo-siCX3CL1 was administered intravenously every 3 days. Either 5 or 12 days after treatment, mice were sacrificed and tumor samples were taken, measured, weighed, and used for further analyses.

Blood GLuc assay. In order to monitor SL4 tumor size in the orthotopic implantation model, blood GLuc activity was measured as described previously $(73,74)$. In short, the SL4-GLuc cell line was established by transduction of lentivirus encoding the GLuc gene and the cells were implanted orthotopically. Blood was collected from the tumor-bearing mice twice a week, and blood GLuc activity was measured using a GloMax 96 Microplate Luminometer (Promega).

Ultrasound imaging. Ultrasound imaging was performed to measure tumor size twice a week until the end of the study using the Vevo 2100 System (VisualSonic) with MS550S probe (frequency $40 \mathrm{MHz}$ ). Tumor-bearing mice were anesthetized with intraperitoneal injection of ketamine $(100 \mathrm{mg} / \mathrm{kg})$ and xylazine $(10 \mathrm{mg} / \mathrm{kg})$ and secured to a heated platform. Abdominal hair was removed, and the exposed skin was covered with ultrasound gel. The probe was applied to the skin, and images were acquired. Tumor tissue in abdominal cavity was identified as a low echoic mass on ultrasound image. The long diameter (LD) and short diameter (SD) were measured. Tumor volume was calculated using the following formula: tumor volume $=\left(L D \times S D^{2}\right) / 2$.

Statistics. The measured values are presented as mean \pm SEM. Comparison between groups was made using ANOVA with HolmŠ́dák post-hoc test. Two-tailed $t$ tests were used between data comparing only 2 groups. Statistical significance was defined as $P<0.05$.

Study approval. All animal procedures followed US Department of Health \& Human Services Public Health Service on Humane Care of Laboratory Animals guidelines and were approved by the Massachusetts General Hospital Institutional Animal Care and Use Committee.

\section{Author contributions}

KJ, ADL, RL, DGA, RKJ, and DF designed research. KJ, TH, OFK, PSK, JI, NNR, and EC performed research. JWC and CGW contributed reagents. KJ, TH, OFK, ADL, SHY, TPP, RKJ, and DF analyzed data. KJ, SHY, TPP, RKJ, and DF wrote the paper.

\section{Acknowledgments}

This work was supported by National Cancer Institute (NCI) program project grant P01-CA080124 (to RKJ and DF) and also in part by NCI grants R01-CA126642, R35-CA197743 (to RKJ) and R01CA096915 (to DF); the NCI/Federal Share Proton Beam Program Income (to RKJ); NIH DP2 OD008780 and R00 CA137167 (to TPP); Department of Defense W81XWH-10-1-0016 (to RKJ); and an Executive Committee on Research Tosteson Fund for Medical Discovery Fellowship (to KJ). We thank Peigen Huang, John D. Martin, Sylvie Roberge, Carolyn Smith, Tsion Hbatmu, Anna Khachatryan, Shan Chin (Steele Laboratories, MGH, Boston, MA USA), Jennie Zhao and Danny Cao (Wellman Center for Photomedicine, MGH, Boston, MA USA) for experimental assistance, and Hyunsung Park (Harvard School of Engineering and Applied Sciences, Cambridge, MA USA) for technical help in microscopy. We also thank Seong-Ik Hwang (The Boston Dental Hospital, Cheonan, Republic of Korea) for help in illustration.

Address correspondence to: Rakesh K. Jain or Dai Fukumura, 100 Blossom Street, Cox 7, Massachusetts General Hospital, Boston, Massachusetts 02114, USA. Phone: 617.726.8143; Email: jain@ steele.mgh.harvard.edu (R.K. Jain); dai@steele.mgh.harvard.edu (D. Fukumura).

EC's present address is: Department of Medical System Engineering and School of Mechatronics, Gwangju Institute of Science and Technology, Gwangju, Republic of Korea.
1. Jain RK. Antiangiogenesis strategies revisited: from starving tumors to alleviating hypoxia. Cancer Cell. 2014;26(5):605-622.

2. Carmeliet P, Jain RK. Molecular mechanisms and clinical applications of angiogenesis. Nature. 2011;473(7347):298-307.

3. Sitohy B, Nagy JA, Dvorak HF. Anti-VEGF/ VEGFR therapy for cancer: reassessing the tar- get. Cancer Res. 2012;72(8):1909-1914.

4. Weis SM, Cheresh DA. Tumor angiogenesis: molecular pathways and therapeutic targets. Nat Med. 2011;17(11):1359-1370.

5. Ferrara N, Adamis AP. Ten years of anti-vascular endothelial growth factor therapy. Nat Rev Drug Discov. 2016;15(6):385-403.

6. Chung AS, et al. An interleukin-17-mediated paracrine network promotes tumor resistance to anti-angiogenic therapy. Nat Med. 2013;19(9):1114-1123.

7. Hanahan D, Coussens LM. Accessories to the crime: functions of cells recruited to the tumor microenvironment. Cancer Cell. 2012;21(3):309-322.

8. Casazza A, et al. Impeding macrophage entry 
into hypoxic tumor areas by Sema3A/Nrp1 signaling blockade inhibits angiogenesis and restores antitumor immunity. Cancer Cell. 2013;24(6):695-709.

9. Rigamonti N, Kadioglu E, Keklikoglou I, Wyser Rmili C, Leow CC, De Palma M. Role of angiopoietin-2 in adaptive tumor resitance to VEGF signaling blockade. Cell Rep. 2014;8(3):696-706.

10. Noy R, Pollard JW. Tumor-associated macrophages: from mechanisms to therapy. Immunity. 2014;41(1):49-61.

11. Kim C, et al. Vascular RhoJ is an effective and selective target for tumor angiogenesis and vascular disruption. Cancer Cell. 2014;25(1):102-117.

12. Franklin RA, et al. The cellular and molecular origin of tumor-associated macrophages. Science. 2014;344(6186):921-925.

13. Rivera L, Pandika M, Bergers G. Escape mechanisms from antiangiogenic therapy: an immune cell's perspective. Adv Exp Med Biol. 2014;772:83-99.

14. Pardoll DM. The blockade of immune checkpoints in cancer immunotherapy. Nat Rev Cancer. 2012;12(4):252-264.

15. Schmid MC, Varner JA. Myeloid cells in tumor inflammation. Vasc Cell. 2012;4(1):14.

16. Finisguerra $V$, et al. MET is required for the recruitment of anti-tumoural neutrophils. Nature. 2015;522(7556):349-353.

17. Buchbinder EI, Hodi FS. Melanoma in 2015: immune-checkpoint blockade - durable cancer control. Nat Rev Clin Oncol. 2016;13(2):77-78.

18. Baumeister SH, Freeman GJ, Dranoff G, Sharpe AH. Coinhibitory pathways in immunotherapy for cancer. Annu Rev Immunol. 2016;34:539-573.

19. Topalian SL, Taube JM, Anders RA, Pardoll DM. Mechanism-driven biomarkers to guide immune checkpoint blockade in cancer therapy. Nat Rev Cancer. 2016;16(5):275-287.

20. D'Angelo SP, et al. Efficacy and safety of nivolumab alone or in combination with ipilimumab in patients with mucosal melanoma: a pooled analysis. JClin Oncol. 2017;35(2):226-235.

21. Ratcliffe MJ, et al. Agreement between programmed cell death ligand-1 diagnostic assays across multiple protein expression cut-offs in nonsmall cell lung cancer [published online ahead of print January 10, 2017]. Clin Cancer Res. https:// doi.org/10.1158/1078-0432.CCR-16-2375.

22. Kim JE, et al. Combination therapy with antiPD-1, anti-TIM-3, and focal radiation results in regression of murine gliomas. Clin Cancer Res. 2017;23(1):124-136.

23. Shojaei F, et al. Tumor refractoriness to anti-VEGF treatment is mediated by CD11b+Gr1+ myeloid cells. Nat Biotechnol. 2007;25(8):911-920.

24. Rivera LB, Meyronet D, Hervieu V, Frederick MJ, Bergsland E, Bergers G. Intratumoral myeloid cells regulate responsiveness and resistance to antiangiogenic therapy. Cell Rep. 2015;11(4):577-591.

25. Talmadge JE, Gabrilovich DI. History of myeloid-derived suppressor cells. Nat Rev Cancer. 2013;13(10):739-752.

26. Kumar V, Patel S, Tcyganov E, Gabrilovich DI. The nature of myeloid-derived suppressor cells in the tumor microenvironment. Trends Immunol. 2016;37(3):208-220.
27. Bronte V, et al. Recommendations for myeloidderived suppressor cell nomenclature and characterization standards. Nat Commun. 2016;7:12150

28. Peranzoni E, et al. Myeloid-derived suppressor cell heterogeneity and subset definition. Curr Opin Immunol. 2010;22(2):238-244.

29. Movahedi K, et al. Identification of discrete tumor-induced myeloid-derived suppressor cell subpopulations with distinct $\mathrm{T}$ cell-suppressive activity. Blood. 2008;111(8):4233-4244.

30. Youn JI, Nagaraj S, Collazo M, Gabrilovich DI. Subsets of myeloid-derived suppressor cells in tumor-bearing mice. J Immunol. 2008;181(8):5791-5802.

31. Fong AM, et al. Fractalkine and CX3CR1 mediate a novel mechanism of leukocyte capture, firm adhesion, and activation under physiologic flow. JExp Med.1998;188(8):1413-1419.

32. Nahrendorf $\mathrm{M}$, et al. The healing myocardium sequentially mobilizes two monocyte subsets with divergent and complementary functions. JExp Med. 2007;204(12):3037-3047.

33. Kim P, et al. In vivo wide-area cellular imaging by side-view endomicroscopy. Nat Methods. 2010;7(4):303-305.

34. Tong RT, Boucher Y, Kozin SV, Winkler F, Hicklin DJ, Jain RK. Vascular normalization by vascular endothelial growth factor receptor 2 blockade induces a pressure gradient across the vasculature and improves drug penetration in tumors. Cancer Res. 2004;64(11):3731-3736.

35. Highfill SL, et al. Disruption of CXCR2-mediated MDSC tumor trafficking enhances anti-PD1 efficacy. Sci Transl Med. 2014;6(237):237ra67.

36. Ali K, et al. Inactivation of PI(3) K p $110 \delta$ breaks regulatory T-cell-mediated immune tolerance to cancer. Nature. 2014;510(7505):407-411.

37. Ries $\mathrm{CH}$, et al. Targeting tumor-associated macrophages with anti-CSF-1R antibody reveals a strategy for cancer therapy. Cancer Cell. 2014;25(6):846-859.

38. Stromnes IM, et al. Targeted depletion of an MDSC subset unmasks pancreatic ductal adenocarcinoma to adaptive immunity. Gut. 2014;63(11):1769-1781.

39. Qin H, et al. Generation of a new therapeutic peptide that depletes myeloid-derived suppressor cells in tumor-bearing mice. Nat Med. 2014;20(6):676-681.

40. Katoh H, Wang D, Daikoku T, Sun H, Dey SK, Dubois RN. CXCR2-expressing myeloidderived suppressor cells are essential to promote colitis-associated tumorigenesis. Cancer Cell. 2013;24(5):631-644.

41. Kim K, et al. Eradication of metastatic mouse cancers resistant to immune checkpoint blockade by suppression of myeloid-derived cells. Proc Natl Acad Sci U S A. 2014;111(32):11774-11779.

42. Di Mitri D, et al. Tumour-infiltrating Gr-1+ myeloid cells antagonize senescence in cancer. Nature. 2014;515(7525):134-137.

43. Damuzzo V, et al. Complexity and challenges in defining myeloid-derived suppressor cells. Cytometry B Clin Cytom. 2015;88(2):77-91.

44. Gabrilovich DI, Ostrand-Rosenberg S, Bronte V. Coordinated regulation of myeloid cells by tumours. Nat Rev Immunol. 2012;12(4):253-268.

45. Ritsma L, Steller EJ, Ellenbroek SI, Kranenburg
O, Borel Rinkes IH, van Rheenen J. Surgical implantation of an abdominal imaging window for intravital microscopy. Nat Protoc. 2013;8(3):583-594.

46. Palmer GM, et al. In vivo optical molecular imaging and analysis in mice using dorsal window chamber models applied to hypoxia, vasculature and fluorescent reporters. Nat Protoc. 2011;6(9):1355-1366.

47. Kirkpatrick ND, et al. Video-rate resonant scanning multiphoton microscopy: An emerging technique for intravital imaging of the tumor microenvironment. Intravital. 2012;1(1):intv.21557.

48. Auffray C, et al. Monitoring of blood vessels and tissues by a population of monocytes with patrolling behavior. Science. 2007;317(5838):666-670.

49. Carlin LM, et al. Nr4a1-dependent Ly6C(low) monocytes monitor endothelial cells and orchestrate their disposal. Cell. 2013;153(2):362-375.

50. Saja MF, et al. Triglyceride-rich lipoproteins modulate the distribution and extravasation of Ly6C/Gr1(low) monocytes. Cell Rep. 2015;12(11):1802-1815.

51. Jung $\mathrm{S}$, et al. Analysis of fractalkine receptor CX(3)CR1 function by targeted deletion and green fluorescent protein reporter gene insertion. Mol Cell Biol. 2000;20(11):4106-4114.

52. Jung K, et al. Endoscopic time-lapse imaging of immune cells in infarcted mouse hearts. Circ Res. 2013;112(6):891-899.

53. Haskell CA, Cleary MD, Charo IF. Molecular uncoupling of fractalkine-mediated cell adhesion and signal transduction. Rapid flow arrest of CX3CR1-expressing cells is independent of G-protein activation. J Biol Chem. 1999;274(15):10053-10058.

54. Landsman L, et al. CX3CR1 is required for monocyte homeostasis and atherogenesis by promoting cell survival. Blood. 2009;113(4):963-972.

55. Kim KW, et al. In vivo structure/function and expression analysis of the $\mathrm{CX} 3 \mathrm{C}$ chemokine fractalkine. Blood. 2011;118(22):e156-e167.

56. Willett CG, et al. Efficacy, safety, and biomarkers of neoadjuvant bevacizumab, radiation therapy, and fluorouracil in rectal cancer: a multidisciplinary phase II study. JClin Oncol. 2009;27(18):3020-3026.

57. Xu L, et al. Direct evidence that bevacizumab, an anti-VEGF antibody, up-regulates SDF1alpha, CXCR4, CXCL6, and neuropilin 1 in tumors from patients with rectal cancer. Cancer Res. 2009;69(20):7905-7910.

58. Geissmann F, Jung S, Littman DR. Blood monocytes consist of two principal subsets with distinct migratory properties. Immunity. 2003;19(1):71-82.

59. Qian BZ, et al. CCL2 recruits inflammatory monocytes to facilitate breast-tumour metastasis. Nature. 2011;475(7355):222-225.

60. Dahlman JE, et al. In vivo endothelial siRNA delivery using polymeric nanoparticles with low molecular weight. Nat Nanotechnol. 2014;9(8):648-655.

61. Morari J, et al. Fractalkine (CX3CL1) is involved in the early activation of hypothalamic inflammation in experimental obesity. Diabetes. 2014;63(11):3770-3784.

62. Yang L, et al. Abrogation of TGF beta signaling in mammary carcinomas recruits $\mathrm{Gr}-1+\mathrm{CD} 11 \mathrm{~b}+$ 
myeloid cells that promote metastasis. Cancer Cell. 2008;13(1):23-35.

63. Huang $\mathrm{Y}$, et al. Vascular normalizing doses of antiangiogenic treatment reprogram the immunosuppressive tumor microenvironment and enhance immunotherapy. Proc Natl Acad Sci U S A. 2012;109(43):17561-17566.

64. Pernot $S$, et al. Colorectal cancer and immunity: what we know and perspectives. World J Gastroenterol. 2014;20(14):3738-3750.

65 . Hu YL, et al. Hypoxia-induced autophagy promotes tumor cell survival and adaptation to antiangiogenic treatment in glioblastoma. Cancer Res. 2012;72(7):1773-1783.

66. Milosevic MF, et al. Sorafenib increases tumor hypoxia in cervical cancer patients treated with radiation therapy: results of a phase 1 clinical study. Int J Radiat Oncol Biol Phys. 2016;94(1):111-117.

67. Chiu DK, et al. Hypoxia induces myeloid-derived suppressor cell recruitment to hepatocellular carcinoma through chemokine ( $\mathrm{C}-\mathrm{C}$ motif) ligand 26. Hepatology. 2016;64(3):797-813.

68. Rivera LB, Bergers G. Intertwined regulation of angiogenesis and immunity by myeloid cells. Trends Immunol. 2015;36(4):240-249.

69. Rahbari NN, et al. Anti-VEGF therapy induces ECM remodeling and mechanical barriers to therapy in colorectal cancer liver metastases. Sci Transl Med. 2016;8(360):360ra135.

70. Hanna RN, et al. Patrolling monocytes control tumor metastasis to the lung. Science. 2015;350(6263):985-990.

71. Morimoto-Tomita M, Ohashi Y, Matsubara A,
Tsuiji M, Irimura T. Mouse colon carcinoma cells established for high incidence of experimental hepatic metastasis exhibit accelerated and anchorage-independent growth. Clin Exp Metastasis. 2005;22(6):513-521.

72. Zhang Y, Davis C, Ryan J, Janney C, Peña MM. Development and characterization of a reliable mouse model of colorectal cancer metastasis to the liver. Clin Exp Metastasis. 2013;30(7):903-918.

73. Chung E, Yamashita H, Au P, Tannous BA, Fukumura D, Jain RK. Secreted Gaussia luciferase as a biomarker for monitoring tumor progression and treatment response of systemic metastases. PLoS One. 2009;4(12):e8316.

74. Tannous BA. Gaussia luciferase reporter assay for monitoring biological processes in culture and in vivo. Nat Protoc. 2009;4(4):582-591. 